\title{
Shock-Wave Solutions of the Einstein Equations: The Oppenheimer-Snyder Model of Gravitational Collapse Extended to the Case of Non-Zero Pressure
}

\author{
Joel Smoller \& Blake Temple
}

Communicated by T.-P. LIU

\section{Introduction}

In their classic 1939 paper, OPPENHEIMER \& SNYDER [9] introduced the first mathematical model for gravitational collapse of stars based on spherically symmetric solutions of the Einstein gravitational field equations. In this pioneering paper, OPPENHEIMER \& SNYDER gave the first rigorous results describing gravitational collapse of stellar objects, and the remarkable conclusion of this work was that "black holes" could form from gravitational collapse in massive stars. In his comprehensive article on the history of the subject of gravitation [4, page 226, paragraph 4] ISRAEL references the Oppenheimer-Snyder paper as having strong claims to be considered the most daring and uncannily prophetic paper ever published in the field. Indeed, the paper appeared a quarter of a century before the process of gravitational collapse was widely accepted as the explanation for a variety of astronomical events. The Oppenheimer-Snyder paper also provided the first example in which a solution of the Einstein equations having interesting dynamics was constructed by using the covariance of the equations to match two simpler solutions across an interface. However, it is well known that the Oppenheimer-Snyder model requires the simplifying assumption that the pressure be identically zero. In this paper we obtain a generalization of the Oppenheimer-Snyder model describing gravitational collapse which extends their model to the case when the pressure is non-zero. Our idea is to treat the case $p \neq 0$ by replacing the boundary surface of the star in the OppenheimerSnyder model by a shock-wave interface across which mass and momentum are transported. In the limit $p=0$ we obtain the Oppenheimer-Snyder solution, and in this limit we observe that the interface reduces to what is referred to as a contact discontinuity in the mathematical theory of shock waves, a degenerate discontinuous solution in which neither mass nor momentum crosses the interface $[1,5,11]$. 
The Oppenheimer-Snyder model was constructed by matching two particular solutions of the Einstein field equations

$$
G=\kappa T
$$

across an interface which represents the boundary of a star, and in Section 3 of this paper we present a general theory for matching two solutions of the Einstein field equations at arbitrary shock-wave interfaces across which the metric $g$ is only Lipschitz continuous, i.e., smooth surfaces across which the first derivatives of the metric suffer at worst a jump discontinuity. Here $G$ denotes the Einstein curvature tensor which is determined by $g$ and derivatives of $g$ up to second order, and $T$ denotes the stress-energy tensor, the source of the gravitational field. In Sections 4 and 5 we apply this general theory to explicitly construct shock-wave interfaces in spherically symmetric solutions of the Einstein equations (1.1). These provide a natural generalization of the Oppenheimer-Snyder model to the case of non-zero pressure.

The general theory in Section 3 is based on formulas first derived by IsRAEL [3] (see also [8]) which relate the jump in the second fundamental form across a shock surface to the Rankine-Hugoniot jump relations for conservation across the surface. We give a reasonably self-contained development of this theory (Section 3, Theorem 2), and we apply it in later sections to derive conditions for conservation across shock surfaces that generalize the Oppenheimer-Snyder case. One of our purposes in Section 3 is to make the theory of shock waves in general relativity readily accessible to researchers in the field of nonlinear hyperbolic conservation laws. Indeed, this subject, which is referenced by the heading junction conditions in [8], has received little notice since the early sixties, and is not even mentioned in most introductory texts on the subject, e.g., WeINBERG [15]. Our second purpose is to clarify the physical significance of Lipschitz continuous shock surfaces, and to derive conditions under which delta-function singularities can appear in components $G_{i j}$ when the Einstein equations are interpreted in the weak sense.

Our interest here is motivated by the observation that there is a remarkable simplification that occurs in the Einstein equations over the classical Euler equations which form a subsystem of (1.1) when $T$ is taken to be the stress-energy tensor for a perfect fluid, and we are interested in pursuing the idea that this may produce computational advantages for shock waves. The point is that in calculating solutions of the classical Euler equations $\operatorname{div} T=0$, one must deal with differencing discontinuous functions in $T$ (the fluid density $\rho$, pressure $p$ and velocity $\boldsymbol{u}$ ), when shock waves form, and this generates the unpleasant Gibbs-type oscillations in approximate solutions generated by finite-difference schemes. But in solving $G=\kappa T$, the Euler equations follow as a subsystem due to the fact that the Einstein curvature tensor $G$ satisfies $\operatorname{div} G=0$ identically as a consequence of the Bianchi identities of geometry. Said differently, since (1.1) does not involve derivatives of $T$, using (1.1) one can solve shock wave problems without ever taking a derivative of the fluid variables in $T$, i.e., the variables $(\rho, \boldsymbol{u}, p)$ that become discontinuous and non-differentiable at the shock. Now $G$ involves second derivatives of the metric potentials $g_{i j}$, so it is natural to ask whether the difficulties in solving $\operatorname{div} T=0$ when $T$ becomes discontinuous at shock waves is replaced by corresponding 
difficulties in the Einstein equations due to the formation of discontinuities in the first derivatives of the metric components $g_{i j}$. Theorem 4 of Section 3 gives a sense in which this is not the case. Indeed, in Theorem 4 we show that the Einstein tensor $G$, and hence the stress tensor $T$ itself, are free of delta-function sources at a shock in any coordinate system if and only if there exists some coordinate system in a neighborhood of the shock in which the metric potentials are continuously differentiable functions of these coordinates, with Lipschitz continuous first derivatives (i.e., $C^{1,1}$ functions). Thus, in numerically simulating the second derivatives that appear in the Einstein tensor $G$ when we solve $G=\kappa T$, we need only difference Lipschitz continuous functions at a shock wave, while in numerically simulating $\operatorname{div} T=0$, we must difference discontinuous functions and deal with the disturbing Gibbs-type oscillations that appear in the approximate solutions. ${ }^{1}$

The advantage of having the Euler equations $\operatorname{div} T=0$ as identities in the Einstein equations also is essential in our construction of the Oppenheimer-Snyder shock-wave solutions in Sections 4 and 5. In this setting, the presence of the metric potentials $g_{i j}$ allows us to solve the problem by first matching the metric across the shock, and this gives us directly an explicit formula for the shock position without requiring that we solve the implicit jump conditions (the weak form of $\operatorname{div} T=0$ ) for the fluids across the shock. Moreover, after the matching is done, the jump conditions reduce in complexity from two to one nontrivial constraint. This reduction of the jump conditions after the metrics are matched represents a constraint on the weak solutions of $\operatorname{div} T=0$, which follows because $\operatorname{div} T=0$ is an identity on solutions of $G=\kappa T$.

As another corollary of the results in Section 3 we also show (Theorem 1 below) that a weak solution of $R_{i j}=0$ that contains a Lipschitz continuous shock surface $\Sigma$ is equivalent (under a singular coordinate transformation) to a metric that is smooth across $\Sigma$. This gives a sense in which shock waves can only arise as coordinate anomalies in the source-free Einstein equations.

We now briefly discuss the issues that arise in the general theory of Lipschitz continuous shock waves for the Einstein equations, and the relevance of this theory to our generalization of the Oppenheimer-Snyder problem. IsRAEL [3] was the first to derive the formulas that express the jump conditions

$$
\left[G_{j}^{i}\right] n_{i}=0
$$

across a shock interface in terms of the second fundamental form $K$ (the extrinsic curvature) of the surface, $K$ being determined separately on each side of the interface. Throughout this paper, $[f]$ denotes the jump in the quantity $f$ across a shock interface. The $n_{i}$ denote the components of the normal vector to $\Sigma$. In light

\footnotetext{
${ }^{1}$ The gravitational potentials $g_{i j}$ play a role similar to that of the vector potential $A$ in the theory of electro-magnetism. In the latter, choosing $F=d A$ has the effect of making the Maxwell equations $d F=0$ hold automatically, in the same way that choosing $G=\kappa T$ has the effect of making the Euler equations $\operatorname{div} T=0$ hold automatically.
} 
of the Einstein equations (1.1), equation (1.2) is equivalent to the RankineHugoniot jump relations [12]

$$
\left[T_{j}^{i}\right] n_{i}=0 .
$$

The result (due to IsRael [3] — we reprove it here for completeness) is that (1.2) holds if and only if the jump in $K$ across the surface satisfies what we refer to as the Israel equations (cf. equations (2.11), (2.12)):

$$
\begin{aligned}
{\left[\operatorname{tr}\left(K^{2}\right)-(\operatorname{tr} K)^{2}\right] } & =0, \\
{[\operatorname{div} K-d(\operatorname{tr} K)] } & =0 .
\end{aligned}
$$

(Here, tr denotes the trace and $d$ denotes exterior differentiation restricted to the surface $\Sigma$.) ISRAEL used this to show that if $\Sigma$ is a smooth, non-null hypersurface across which the metric components are only Lipschitz continuous, but arbitrarily smooth on either side (we always assume that all derivatives are continuous up to the boundary $\Sigma$ from either side of $\Sigma$, and we refer to such a surface as a Lipschitz continuous shock wave), then a necessary and sufficient condition for the surface to be coordinate transformable to a surface across which the derivatives of the metric are continuous is the condition that the second fundamental form be continuous across $\Sigma$, a coordinate invariant condition. It then follows from (1.4) that the weak form (1.3) of conservation also holds when $K$ is continuous across $\Sigma$. Note that the jump in the derivatives of the metric cannot be transformed away by smooth coordinate transformations. The idea here is to allow $C^{1,1}$ coordinate transformations, (transformations with Lipschitz continuous first derivatives), i.e., those smooth enough to preserve the tensor transformation laws, but weak enough to allow jumps in the second derivatives of the transformation in order to adjust the jumps in the first derivatives of the metric tensor across $\Sigma$. In this paper we show that $C^{1,1}$ is natural for the formulation of weak derivatives in the curvature tensor. In Section 3 we show that delta-function sources in the Einstein tensor $G$ (which involves second derivatives of the metric tensor) exist at a point $P \in \Sigma$ if and only if $[K] \neq 0$ at $P$ (cf. [8]). Moreover, we show that the existence of delta-function sources in $G$ on $\Sigma$ for an arbitrary Lipschitz continuous shock wave has a covariant meaning in the sense that the existence or non-existence of delta-function sources in $G$ is independent of coordinates when we restrict to $C^{1,1}$ coordinate transformations. Thus we conclude in general that $G$ is free of delta-function sources on a Lipschitz continuous shock surface if and only if $[K]=0$. It remains to give an interpretation of the jump conditions (1.3) when there are delta-function sources in $G$ (and hence in the stress tensor $T$ ) on a Lipschitz continuous shock surface $\Sigma$. When $[K]=0$, and hence there are no delta function sources on the surface, the metric is coordinate transformable to a $C^{1}$ metric, and thus it is straightforward to show that there exist "locally Lorentzian" coordinate frames in a neighborhood of each point $P \in \Sigma$, coordinates in which $g_{i j}=\eta_{i j} \equiv \operatorname{diag}\{-1,1,1,1\}$ and $g_{i j, k}=0$ at $P$. In such coordinates one can show that the jump conditions (1.3) express local conservation of energy and momentum, i.e., exact conservation to within errors due to the perturbation from flat space caused by the non-vanishing curvature. It is important to note that it is the global, integral formulation of conservation of 
energy and momentum that has a fundamental physical interpretation in flat space, and such integral conservation laws do not hold globally, but can be localized, when curvature is present. The physical justification for the localization is based on the existence of locally Lorentzian coordinate frames. When $[K] \neq 0$ at a point $P \in \Sigma$, we show that there do not exist coordinate transformations within the class of $C^{1,1}$ mappings that take the given coordinates to a locally Lorentzian coordinate frame at $P$. As a final comment, consider the case of a Lipschitz continuous shock surface that does not satisfy $[K]=0$, so that there are delta-function sources in $G$ and $T$ on the surface, but such that conservation in the form of the jump conditions (1.3) does hold across the surface. (An example of such a shock is given in the last paragraph of Section 3.) Then in terms of the fluid flowing across the surface, the surface is not acting as a source of momentum or energy. Thus, in a sense, the delta-function sources of energy and momentum in $T$ are not affecting the fluid flow directly, but are only affecting the gravitational field, i.e., the space-time metric that connects up continuously at the shock. It remains an open question whether such Lipschitz continuous shock waves can appear in the time dynamics of the Einstein equations after the fluid variables shock. We show in Section 5 that in the case of spherically symmetric solutions (the case that applies to the Oppenheimer-Snyder problem), all conservative shock wave solutions are coordinate-transformable to metrics that are in $C^{1}$ across the shock because, in the presence of spherical symmetry, the jump conditions (1.3) imply that $[K]=0$ (cf. [3] on this point). Thus, in particular, $G$ contains no delta-function sources on spherically symmetric shock wave solutions of the Einstein equations.

As a corollary of the results in Section 3 we prove Theorem 1 below, which states that shock-wave-type singularities across which $g$ remains Lipschitz continuous cannot form in weak solutions of the source-free Einstein equations $R_{i j}=0$ or $G_{i j}=0$. Thus, if we view $R_{i j}\left(g_{i j}\right)=0$ or $G_{i j}\left(g_{i j}\right)$ as a second-order hyperbolic partial differential equation in the metric components $g_{i j}$, then a natural question arises as to what type of singularities can form in solutions starting from smooth initial data on some space-like hypersurface. For example, the equations $R_{i j}=0$ or $G_{i j}=0$ can be written as first-order quasi-linear partial differential equations in $g$ and derivatives of $g$, and the theory of shock waves tells us that in general such non-linear partial differential equations form shock-wave discontinuities in finite time [1, 11]. The following result, which is an immediate consequence of Corollary 5 of Section 3 , partially validates the statement that such shock-wave singularities in $R_{i j}=0$ or $G_{i j}=0$ are only coordinate anomalies, and can be transformed away by coordinate transformation:

Theorem 1. If a smooth shock surface $\Sigma$ forms in weak solutions of $R_{\alpha \beta}=0$ or $G_{\alpha \beta}=0$ posed in some given coordinate system $y$, such that the $y$-components $g_{\alpha \beta}$ of the metric tensor $g$ are Lipschitz continuous across $\Sigma$, and are $C^{k}$ functions of $y$ on either side of $\Sigma$ (continuous up to the boundary on either side separately), then there exists a regular $C^{1,1}$ coordinate transformation taking $y \rightarrow x$, such that the components $g_{i j}$ of $g$ in $x$-coordinates are actually $C^{k}$ functions of $x$ in a neighborhood of each point on the surface $\Sigma$. 
It is important at this point to discuss carefully our contributions here in the context of the important earlier work of IsRaEL [3] as expounded in MisNer, ThORNE \& WheELER [8]. The treatment in [8] is based upon arguments due to ISRAEL which relate the jump conditions for conservation to the jump in the second fundamental form across a shock surface. The main conclusion of ISRAEL is that there exists a coordinate system in which the metric is of class $C^{1}$ across a shock if and only if the jump in the second fundamental form $K$ vanishes at the shock. Misner, THORNe \& WheELer argue that the vanishing of the jump in $K$ across a shock surface is also equivalent to the absence of delta function sources in the fluid variables at the shock. Their analysis is based on the construction of Gaussian normal coordinate systems, the coordinate systems in which the metric is in $C^{1}$ whenever the jump in $K$ vanishes across the shock. Their goal is to be physically intuitive, and as a result their arguments seem to us to be mathematically incomplete. In particular, this comment applies to their derivation of the important conclusion (equation 21.167, page 553 of their book) which reads

$$
\text { absence of surface layers } \Leftrightarrow \text { "continuity" of } g_{i j} \text { and } K_{i j} \text {. }
$$

Furthermore, the sense in which this equation has a coordinate-independent meaning is not made clear. Our point of view is that the natural notion of "continuity" (their quotes) is Lipschitz continuity, and the natural class of coordinate transformations is $C^{1,1}$. In fact, we show that a mathematically rigorous formulation of (1.6) is the statement: "The absence of surface layers is equivalent to the continuity of $K$, in the presence of Lipschitz continuity of the metric." In our development we are led naturally to classes of coordinate frames that are related to each other by coordinate transformations that are precisely of class $C^{1,1}$ in a neighborhood of a shock surface. This is important because we use the fact that this is the weakest smoothness class for test functions that leads to a consistent weak formulation of the curvature tensor. Using distribution theory, we prove that $G$ is free of delta-function sources at any Lipschitz continuous matching of the metrics, if and only if $K$ is continuous across the shock, which holds if and only if there exists a coordinate system (the Gaussian normal coordinates) which is related to the original coordinates by a $C^{1,1}$ coordinate transformation, such that in this new coordinate system, the metric is of class $C^{1,1}$ across the shock (see Theorem 4). We believe that these are not just technical points. Indeed, several interesting new results emerge once we identify the natural smoothness class of the metrics and the natural smoothness class of the coordinate transformations at the shock. In particular, we need this extra rigor in order to prove that the above equivalencies are also equivalent to the existence of locally Lorentzian frames on the surfaces which can be reached within the class of $C^{1,1}$ coordinate transformations. In Theorem 1 we also show the sense in which shock waves cannot form in the source-free Einstein equations, $G=0$. This result seems to have been overlooked in other treatments of surface layers, e.g., [3]. Our analysis also leads us to the interesting conclusion that conservation alone implies no delta-function sources on surfaces for spherically symmetric metrics which match Lipschitz continuously across a shock surface. (This result is of fundamental importance in our generalization of the Oppenheimer-Snyder construction.) As a third application of our 
analysis, we also obtain the result that the (Ricci) scalar curvature never has delta-function sources at any Lipschitz continuous shock wave.

In conclusion, we believe that we have discovered some new results here, but we wish to make it clear that our analysis of general Lipschitz continuous shock waves is based essentially upon ISRAEL's important paper [3].

In Section 4 we begin our discussion of the generalization of the Oppenheimer-Snyder model to the case of non-zero pressure. Our procedure is to first match the Friedmann-Robertson-Walker-type metrics (which we refer to as R-W metrics, cf. [15, p. 412]) to the Oppenheimer-Tolman-type ${ }^{2}$ metrics (O-T metrics) in a Lipschitz continuous fashion across a shock interface. Here our method is quite general in that we allow arbitrary equations of state on both sides of the shock interface. We also derive a global principle of conservation of mass for these solutions in general. However, such a matching does not ensure that local conservation of energy and momentum (1.2) holds across the shock interface. Thus, in order to ensure the physical conservation requirements, we must apply the general theory of Section 3 to impose the further constraint that the jump in the second fundamental form on the surface be continuous at the shock interface. In the case of spherical symmetry, the one dealt with in this application, the jump conditions impose two independent constraints, but the condition on the second fundamental form reduces these two conditions to a single constraint on the shock surface. This means that the Lipschitz continuity of the metric across the shock reduces by one the number of constraints that must be met to guarantee conservation of energy and momentum across the shock, and this indicates the advantage of introducing the gravitational potential into the problem of constructing fluid-dynamical shock waves in multi-dimensions. In order to meet the remaining constraint of conservation, we obtain ordinary differential equations for the shock surface in which the pressure on one side of the shock is not determined by an equation of state, but rather is one of the unknowns in the system of equations. We end the section by deriving an autonomous system of two ordinary differential equations $((5.35)$ and (5.46)) in two unknowns that simultaneously determine the pressure and the shock surface, and we conclude that one need only solve these in order to obtain shock-wave solutions across which local conservation holds. The global dynamics of the shock surface, its stability, and the behavior of the pressure jump in the large, will be addressed in a future paper.

It is interesting to comment that the problem of checking the jump conditions (1.3) across an interface can be difficult to do directly in a given application. To understand the apparent simplification in constructing shock waves in the setting of general relativity vs. classical fluids, we note that the inclusion of the

\footnotetext{
${ }^{2}$ We choose this as a name for static, spherically symmetric metrics that satisfy the Einstein equations for a perfect fluid. It appears that $\mathrm{O}-\mathrm{T}$ solutions have not been given a name in the literature, and we consider this name to be oppropriate; cf. [14]. The case when the equation of state is of the form energy density = const. is referred to as the interior Schwarzschild metric.
} 
gravitational potential in the fluid equations allows for the introduction of the curvature tensor $G_{i j}$, which always being (identically!) divergence-free, guarantees the conservation laws $\operatorname{div} T=0$ automatically on solutions of the Einstein equations, $G=\kappa T .^{3}$ As we are not aware of any similar formulas for spherically symmetric shock waves in classical conservation laws, we pose the question as to whether the limits $\kappa \rightarrow 0$ and $c \rightarrow \infty$ in $G=\kappa T$ might help in the classical problem of constructing shock waves in fluids. This will be considered in future publications.

Thus, to summarize, our point of view is that the reduction of the jump conditions (1.3) on solutions of (1.1) to covariant conditions on the second fundamental form of the shock surface validates the general method of constructing solutions of the Einstein field equations by matching one simple solution to another (written in a different coordinate system) across a shock interface. This establishes a procedure by which solutions with interesting dynamics can be constructed out of simpler solutions, the interesting dynamics arising because the matched solution dynamically evolves from one solution to a coordinate transformation of the other as the shock surface propagates. We interpret this procedure as a way of bringing the power and covariance of Riemannian geometry to bear upon the problem of constructing shock wave interfaces for fluids in multidimensions.

The plan of this paper is as follows: in Section 2, we give a quick introduction to the Einstein equations, together with some background material. In Section 3, we derive the necessary and sufficient conditions on $K$ in order that (1.2) hold across a shock surface. In Section 4 we introduce the Robertson-Walker and the Oppenheimer-Tolman metrics, and derive a generalization of the Oppenheimer-Snyder model by matching these solutions in a Lipschitz continuous manner across a shock-wave interface. This is accomplished for arbitrary equations of state. In Section 5 we apply the results of Section 3 in order to derive a system of differential equations for the Robertson-Walker pressure so that local conservation holds across the shock. In Appendix i we discuss Gaussian normal coordinates, and Appendix ii is devoted to the derivation of several important identities needed in the matching of the Robertson-Walker and OppenheimerTolman metrics.

\section{Preliminaries}

We consider a four dimensional space-time manifold with metric tensor $g$ having signature $\eta_{i j}=\operatorname{diag}(-1,1,1,1)$. The Einstein field equations (1.1) represent ten equations for the unknown gravitational field, which by definition, is the metric tensor. In a given coordinate system $x=\left(x^{0}, \ldots, x^{3}\right): M \rightarrow R^{4}$ on space-time $M$,

\footnotetext{
${ }^{3}$ Moreover, the curvature tensor $G_{i j}$ involves second derivatives, yet being a tensor, it transforms under the same transformation rules as the undifferentiated metric tensor.
} 
the metric tensor has components $g_{i j}=g_{i j}(x)$, which defines a $4 \times 4$ symmetric matrix at each point. ${ }^{4}$ Here, $x^{0}=c t$, where $c$ is the speed of light.

The Einstein equations (1.1) in a given coordinate system $x$ take the form (see $[13,15])$

$$
G_{i j}=\kappa T_{i j}
$$

where

$$
G_{i j} \equiv R_{i j}-\frac{1}{2} R g_{i j}
$$

is the Einstein curvature tensor, $T_{i j}$ denotes the stress-energy tensor (the source of the gravitational field), $\kappa=8 \pi \mathscr{G} / c^{4}$ where $\mathscr{G}$ is Newton's universal gravitational constant, and $R_{i j}$ and $R$ denote, respectively, the Ricci tensor and the scalar curvature formed from the Riemann curvature tensor of the metric $g$. The Riemann curvature tensor, with components $R_{j k l}^{i}$, is given by

$$
R_{j k l}^{i}=\Gamma_{j k, l}^{i}-\Gamma_{j l, k}^{i}+\Gamma_{\sigma l}^{i} \Gamma_{j k}^{\sigma}-\Gamma_{\sigma k}^{i} \Gamma_{j l}^{\sigma},
$$

and $R_{i j}$ and $R$ are obtained by the contractions

$$
R_{i j}=R_{i \sigma j}^{\sigma}, \quad R=R_{\sigma}^{\sigma} .
$$

Here we use the Einstein summation convention whereby summation is assumed (from 0 to 3) over repeated up-down indices in the same summand of a formula. The notation " $i$ " denotes differentiation with respect to the variable $x^{i}$, and in general (except for Section 3), all indices run from 0 to 3 . The $\Gamma_{j k}^{i}$ denote the Christoffel symbols for the metric connection determined by $g$, and are defined (in $x$-coordinates) by the formulas

$$
\Gamma_{j k}^{i}=\frac{1}{2} g^{\sigma i}\left\{-g_{j k, \sigma}+g_{\sigma j, k}+g_{k \sigma, j}\right\} .
$$

These functions determine the geodesics of the metric $g$, which are by definition solutions of the equations

$$
\frac{d^{2} x^{i}}{d s^{2}}=\Gamma_{j k}^{i} \frac{d x^{j}}{d s} \frac{d x^{k}}{d s}
$$

where $s$ denotes the arc-length parameter. The raising and lowering of the indices is accomplished via the metric tensor. For example,

$$
\begin{aligned}
G_{j}^{i} & =g^{i \sigma} G_{\sigma j}, \\
T_{i j} & =g_{i \sigma} T_{j}^{\sigma},
\end{aligned}
$$

where $g^{i j}$ denotes the inverse of $g_{i j}$.

${ }^{4}$ In Einstein's theory, all physical properties of the gravitational field are determined by the metric tensor. Indeed, the "free-fall" paths through a gravitational field are given by the geodesics of the metric; the "aging time" or "proper time" change for an observer traversing a path through space-time is given by the Minkowski arc length of the path as determined by the metric $g$; and the non-rotating frames carried along geodesic free-fall paths are precisely the frames that are parallel relative to the unique symmetric connection determined by the metric $g$. 
The Einstein tensor $G$ satisfies the condition $\operatorname{div} G=0$, where div denotes the covariant divergence defined in terms of the covariant derivative $\nabla$ of the metric connection for $g$. That is (in components),

$$
\nabla_{k} G_{j}^{i} \equiv G_{j, k}^{i}=G_{j, k}^{i}+\Gamma_{\sigma k}^{i} G_{j}^{\sigma}-\Gamma_{j k}^{\sigma} G_{\sigma}^{i},
$$

so that

$$
(\operatorname{div} G)_{j} \equiv G_{j ; \sigma}^{\sigma}=G_{j, \sigma}^{\sigma}+\Gamma_{\sigma \tau}^{\tau} G_{j}^{\sigma}-\Gamma_{j \tau}^{\sigma} G_{\sigma}^{\tau} .
$$

It is important to note that since $\operatorname{div} G=0$, it follows that for solutions of (1.1) we must have $\operatorname{div} T=0$. The distinction here is that $\operatorname{div} G=0$ is a geometric identity, independent of the Einstein equations, and holds as a consequence of the Bianchi identities, while $\operatorname{div} T=0$ relies on both the identity $\operatorname{div} G=0$ as well as the Einstein equations (1.1). For example, in Section 4, we shall consider the case of a "perfect fluid", wherein the stress-energy tensor takes the form

$$
T_{i j}=\left(p+\rho c^{2}\right) u_{i} u_{j}+p g_{i j} .
$$

In this equation, $p$ denotes the pressure, $u$ denotes the four-velocity of the fluid particle (the velocity of the frame of isotropy of the perfect fluid), and $\rho$ denotes the mass-energy density (which we refer to as the energy density, except when making the analogy with classical fluids, in which case we refer to it as the mass density) of the fluid, as measured in a reference frame moving with the fluid particle. In the case of a barotropic gas, $p$ is assumed to be given by a function of $\rho$ alone: $p=p(\rho)$. Thus in this case, div $T=0$ gives four additional equations which hold on solutions of (1.1). These are four non-linear ${ }^{p}$ equations which reduce to the Euler equations for compressible fluid flow (which express the conservation of energy and momentum) when $g$ is taken to be the flat Minkowski metric $g_{i j} \equiv \eta_{i j}=$ $\operatorname{diag}(-1,1,1,1)$. It is well known that shock wave discontinuities form in solutions of the Euler equations for compressible flow [12]. In the case when shocks form, the Rankine-Hugoniot jump conditions (1.3) express the weak formulation of conservation of energy and momentum across shock surfaces. In Section 4 we generalize the Oppenheimer-Snyder model for gravitational collapse by matching two (metric) solutions of the Einstein equations (1.1) in a Lipschitz continuous manner. We were not able to verify the Rankine-Hugoniot jump relations (1.3) directly because these involve the fluid variables in (2.9), and a direct verification of (1.3) requires using div $T=0$, which is not an identity, and so cannot be managed without invoking the full Einstein equations (1.1). However, in the next section we bypass this problem with a general theorem (cf. [3]) that (1.3) follows as a geometric identity from the corresponding identities $\operatorname{div} G=0$ together with geometrical constraints on the second fundamental form on the shock surface, once one knows that the metric is Lipschitz continuous across the shock surface. The second fundamental form $K: T_{\Sigma} \rightarrow T_{\Sigma}$ on a codimension-one surface $\Sigma$ with normal vector field $\boldsymbol{n}$, imbedded in an ambient Riemannian space with metric tensor $g_{i j}$, is a tensor field defined on the surface in terms of the metric $g$, and describes how the surface is imbedded in the ambient space. Here, $T_{\Sigma}$ denotes the tangent space of $\Sigma$. The second fundamental form $K$ is defined by the condition

$$
K(\boldsymbol{X})=-\nabla_{X} n
$$


for $\boldsymbol{X} \in T_{\Sigma}$. When the metric is only Lipschitz continuous across a codimension-one surface, the second fundamental form $K$ is determined separately from the metric values on either side. In the next section we give necessary and sufficient conditions (the Israel conditions) for conservation to hold at a Lipschitz continuous shockwave interface, the condition being given in terms of geometric conditions on the jump in the second fundamental form across the surface. The conditions are that

$$
\begin{aligned}
& {\left[\operatorname{tr}\left(K^{2}\right)-(\operatorname{tr} K)^{2}\right]=0,} \\
& {[\operatorname{div} K-d(\operatorname{tr} K)]=0,}
\end{aligned}
$$

where tr denotes trace, div denotes covariant divergence, and $d$ denotes exterior differentiation in the surface. We conclude that the physical conservation laws (1.3) turn out to be a consequence of geometrical constraints built a priori into the Einstein tensor, together with geometrical constraints that describe how the shock surface is imbedded in the ambient space-time manifold. We note that a sufficient condition for conservation is that $[K]=0$ everywhere across the surface. In fact, this implies that in Gaussian normal coordinates the metric is then in $C^{1}$ because $K_{i j}=g_{i j, n}$ in these coordinates, where $n$ denotes differentiation in the direction normal to the surface. (See Appendix $i$ and $[3,8,2]$.) As we point out in the next section, the transformation to Gaussian normal coordinates is in general only a $C^{1,1}$ coordinate transformation, but once this transformation is made, the $C^{\infty}$ coordinate transformations alone are sufficient to describe the locally Lorentzian properties of the spacetime. (Recall that by $C^{1,1}$ we mean $C^{1}$ with Lipschitz continuous derivatives.) In the case of metrics that are only Lipschitz continuous, the natural class of coordinate transformations is the class of $C^{1,1}$ transformations. Indeed, if the mapping $x \rightarrow y$ is in $C^{1,1}$, then $\partial x / \partial y$ and $\partial y / \partial x$ are Lipschitz continuous, and thus Lipschitz continuous tensors are mapped to Lipschitz continuous tensors under the mapping $x \rightarrow y$, and this is the least smooth class of transformations that preserves this mapping. Note that by allowing $C^{1,1}$ transformations, we allow derivatives of $\partial x / \partial y$ and $\partial y / \partial x$ to jump, and this allows us to adjust the jump in the derivatives of tensors across a shock surface. For example, if $g=g_{L} \cup g_{R}$, then

$$
g_{i j}=g_{\alpha \beta} \frac{\partial y^{\alpha}}{\partial x^{i}} \frac{\partial y^{\beta}}{\partial x^{j}}
$$

so the jumps in the derivatives of $\partial y^{\alpha} / \partial x^{i}$ change the jumps in the derivatives of $g_{i j}$ across $\Sigma$, and ISRAEL's result states that within the class of $C^{1,1}$ transformations, we can match the derivatives in $g$ across $\Sigma$ if and only if $[K]=0$, the map to Gaussian normal coordinates being in $C^{1,1}$. Now in the Einstein equations $G_{i j}=\kappa T_{i j}, G_{i j}$ is the image of a second-order differential operator on the metric entries $g_{i j}$, and thus in general we expect metrics that are Lipschitz continuous across $\Sigma$ to have delta-function sources in $G$, and hence in the fluid variables $T$, on $\Sigma$. It is natural to ask, first, when do such delta-function sources appear at a shock wave $\Sigma$ given that the metric is only Lipschitz continuous across $\Sigma$, and second, what is the physical significance of such delta-function sources when they do appear? For the first question, we present a proof in the next section that if 
$g=g_{L} \cup g_{R}$ is Lipschitz continuous across $\Sigma$ in a coordinate system $x$, then delta function sources appear in $G$ on $\Sigma$ in $x$-coordinates if and only if $[K] \neq 0$ (cf. [8]). For the physical interpretation of the delta-function sources in $G$, and hence in $T$, when $[K] \neq 0$ at $\Sigma$, we comment that the equivalence of the jump conditions $\left[G_{j}^{i}\right] n_{i}=0=\left[T_{j}^{i}\right] n_{i}$ and the weak formulation of $\operatorname{div} G=0$ at a point $P$ in space-time is based on the existence of locally Lorentzian coordinate frames at $P$; i.e., coordinates in which $g_{i j, k}(P)=0$. In such coordinate frames, space-time is locally flat, and the physical principles of special relativity can thus be identified locally. In particular, the covariant divergence agrees with the classical divergence in locally Lorentzian frames, and the global physical conservation laws $\int_{\partial \Omega}=0$ of special relativity can be reduced in local form to $\operatorname{div} T=0$ in curved spacetime. (It is well known that, except in special cases, there do not exist global conservation laws in general relativity.) In the next section we show that, within the class of $C^{1,1}$ coordinate transformations, there do not exist locally Lorentzian coordinate frames in a neighborhood of a point $P \in \Sigma$ where $G_{i j}$ has a delta-function source. Thus, space-time is not locally flat at points on a Lipschitz continuous shock wave where $G$ has delta-function sources. In Section 5 we show that for spherically symmetric shock waves, $\left[G_{j}^{\sigma}\right] n_{\sigma}=0$ implies $[K]=0$, and thus conservation implies that there are no delta-function sources in the shock waves we construct as generalizations of the Oppenheimer-Snyder case, and thus these solutions are locally Lorentzian at each point on the shock. It is an interesting open question as to whether general Lipschitz continuous shocks can appear in the time evolution of $G=\kappa T$.

It is interesting to note also that in Section 4, when we generalize the Oppenheimer-Snyder solution to arbitrary barotropic equations of state $p=p(\rho)$, we identify a principle of conservation of mass-energy in the large, valid in a physically interesting coordinate system arising quite naturally in the problem.

\section{Lipschitz Continuous Metrics}

In this section we give the proof that the jump conditions (1.2) hold at a Lipschitz continuous shock surface if and only if (2.11) and (2.12) hold. We formulate the theorem in $n$ dimensions for a nonsingular metric $g$ of fixed signature $\eta=\operatorname{diag}\left(\varepsilon_{1}, \ldots, \varepsilon_{n}\right)$ where each $\varepsilon_{i}= \pm 1$. Before stating the theorem, we introduce some notation. Thus let $y \equiv\left(y^{1}, \ldots, y^{n}\right)$ be a smooth coordinate system defined on an $n$-dimensional manifold $M, y: M \rightarrow R^{n}$, and let $\Sigma$ be a smooth hypersurface in $M$. Assume that $\Sigma$ is given locally by $\psi(y)=0$, where $\psi$ is a smooth function satisfying

$$
n_{i} d y^{i} \equiv \frac{\partial \psi}{\partial y^{i}} d y^{i} \neq 0
$$

Let $L$ and $R$ (for "left" and "right") denote the two sides of $M$ defined by the surface $\Sigma$, and let $g_{L}$ and $g_{R}$ denote smooth metrics defined on the left and right side of $\Sigma$, respectively. (It suffices to assume $g_{L}$ and $g_{R}$ are at least in $C^{2}$, with derivatives uniformly bounded at $\Sigma$, and we assume this from here on out.) For completeness, we give a proof of the following theorem due to IsRaEL $[3,8]$. 
Theorem 2. Let $g=g^{L} \cup g^{R}$ denote a non-singular metric of arbitrary signature whose components $g_{i j}$ in $y$-coordinates are smooth on the left and right sides of $\Sigma$, separately, and Lipschitz continuous across the surface. Assume that $\Sigma$ is given locally by $\varphi=0$, where $\varphi$ is smooth, assume that (3.1) holds, and assume that the normal vector $\boldsymbol{n}$ is non-null relative to the metric $g$, so that (without loss of generality) $\boldsymbol{n}$ may be taken to be a unit vector $g_{i j} n^{i} n^{j}=1$. Then

$$
\left[G_{j}^{i}(y(P))\right] n_{i}(y(P))=0
$$

at a point $P \in \Sigma$ if and only if both

$$
\begin{aligned}
{\left[\left(\operatorname{tr} K^{2}\right)-\operatorname{tr}(K)\right] } & =0, \\
{[\operatorname{div} K-d(\operatorname{tr} K)] } & =0,
\end{aligned}
$$

hold. (Here, the invariant operations $\operatorname{div}, \operatorname{tr}$ and $d$ on $K$ are restricted to the surface $\Sigma$.)

Note that by a smooth transformation of the coordinates in a neighborhood of a point $P \in \Sigma$ we may assume that the surface $\Sigma$ is given by $\varphi=y^{n}=0$, so that $\boldsymbol{n}=\partial / \partial y^{n}$. In this case, the invariant conditions (3.3) and (3.4) reduce in $y$-coordinates to

$$
\begin{aligned}
{\left[\left(K_{i}^{i}(y(P))\right)-\left(K_{i}^{i}(y(P))\right)^{2}\right] } & =0, \\
{\left[K_{j ; i}^{i}(y(P))-K_{i, j}^{i}(y(P))\right] } & =0,
\end{aligned}
$$

where the summation in (3.5) and (3.6) is assumed to run from 1 to $n-1$.

The proof of Theorem 2 follows as a consequence of several lemmas. The idea is to construct Gaussian normal coordinates for the surface $\Sigma$, these being coordinates in which the components of the second fundamental form take the simple form $K_{i j}=-\frac{1}{2} g_{i j, n}$. We then use this identity to write the Einstein curvature tensor $G$ and the jump conditions (1.2) in terms of the $K_{i j}$ and obtain (3.5) and (3.6) (cf. $[3,8]$ ). We use the following identities for the components of the curvature tensor $G_{j}^{i}$ in an arbitrary coordinate system:

Lemma 1. The components of $G$ are given by

$$
\begin{aligned}
G_{i}^{i} & =-\sum_{\sigma, \tau \neq i} R_{[\sigma \tau]}^{[\sigma \tau]}, \quad i=1, \ldots, n, \\
G_{j}^{i} & =\sum_{\tau \neq i, j} R_{[j]]}^{[i \tau]}, \quad i \neq j,
\end{aligned}
$$

where the square braces [ ] around a set of indices indicates that summation is to be taken only over the increasing sequences of indices occurring inside the braces.

Proof. To prove (3.7), we have

But

$$
G_{j}^{i}=R_{j}^{i}-\frac{1}{2} R \delta_{j}^{i}
$$

$$
R_{i}^{i}=R_{\tau i}^{\tau i}=\sum_{\tau \neq i} R_{\tau i}^{\tau i}
$$


because $R_{\gamma \delta}^{\alpha \beta}$ is antisymmetric in $(\alpha \beta)$ and $(\gamma \delta)$. Moreover,

$$
R=R_{\sigma \tau}^{\sigma \tau}=2 R_{[\sigma \tau]}^{[\sigma \tau]}
$$

and so

$$
G_{i}^{i}=R_{[\tau i]}^{[\tau i]}-R_{[\sigma \tau]}^{[\sigma \tau]}=-\sum_{\sigma, \tau+i} R^{[\sigma \tau]} .
$$

To prove (3.8) we have

$$
G_{j}^{i}=R_{j}^{i}=R_{\tau j}^{\tau i}=\sum_{\tau \neq i, j} R_{[\tau j]}^{[\tau]]} .
$$

We now construct a Gaussian normal coordinate system $\left(w^{1}, \ldots, w^{n}\right)$ associated with the surface $\Sigma$ in a neighborhood of $P_{0} \in \Sigma$ [2]. To this end, we assume that $g$ has $y$-components $g_{i j}$, and by making a smooth coordinate transformation we may assume without loss of generality that $\Sigma$ is defined (near $P_{0}$ ) by $y^{n}=0$. For each $P \in \Sigma$ let $\gamma_{P}(s)$ denote the geodesic satisfying

$$
\gamma_{P}(0)=P, \quad \dot{\gamma}_{P}(0)=n,
$$

where $\boldsymbol{n}$ is the normal vector to $\Sigma$ at $P, s$ is arc length, and for convenience we assume that $\boldsymbol{n}$ points into the right side of $\Sigma$. We define the $w^{n}$-coordinate in a neighborhood of $P_{0} \in \Sigma$ as the "distance from $\Sigma$ " as follows: if $\gamma_{P}(s)=Q$, then set $w^{n}(Q)=s$. In this way, $w^{n}<0$ on the left side of $\Sigma$, and $w^{n}>0$ on the right side of $\Sigma$. Now define the $w^{i}$-coordinates for $i=1, \ldots, n-1$, by $w^{i}(P)=y^{i}(P)$ for $P \in \Sigma$, and define $w^{i}$ in a neighborhood of $\Sigma$ by taking $w^{i}$ to be constant along each $\gamma_{P}(s)$; i.e.,

$$
w^{i}(Q)=w^{i}(P) \quad \text { if and only if } \quad Q=\gamma_{P}(s)
$$

for some $P$ and $s, i=1, \ldots, n$. The coordinates $w=\left(w^{1}, \ldots, w^{n}\right)$ are called Gaussian normal coordinates in a neighborhood of $P_{0} \in \Sigma$. Note that the Gaussian normal coordinates $w$ are in general only $C^{1,1}$ related to the original $y$-coordinates because the geodesics normal to the surface $\Sigma$ are in general only $C^{1}$ curves since the $\Gamma_{j k}^{i}$ can in general have jump discontinuities at $\Sigma$ when $g$ is only Lipschitz continuous across $\Sigma$. (Indeed, to see this, consider the curves $y_{\bar{y}}(s)$ where $\bar{y}=\left(y^{1}, \ldots, y^{n-1}\right)$ and $(\bar{y}, 0) \in \boldsymbol{R}^{n}$ is the coordinate value of the point $P$ on $\Sigma$ such that $\gamma_{P}(s)=Q$ has $y$-coordinates $y_{\bar{y}}(s)$. Thus, $y(P)=\bar{w}(P)$ for $P \in \Sigma$. But being constructed from families of geodesics on each side of $\Sigma, y_{\bar{y}}(s) \equiv \varphi(\bar{y}, s)$ is a smooth function of $\bar{y}$ and $s$ on each side of $\Sigma$ separately. It remains to check continuity of derivatives at $y^{n}=0$. But, at $s=0$,

$$
\frac{\partial y^{i}}{\partial \bar{w}^{j}}=\frac{\partial y_{\bar{y}}^{i}}{\partial \bar{y}^{j}}(s)=\delta_{j}^{i},
$$

because $y=(\bar{y}, 0)$ at $s=0$. Moreover,

$$
\frac{\partial y^{i}}{\partial \bar{w}^{n}}=\frac{\partial y_{\vec{y}}^{i}}{\partial s}(s)=\boldsymbol{n}^{i},
$$


where $\boldsymbol{n}^{i}$ denote the $y$-coordinates of the normal to $\Sigma$ at $P$. Since the metric is continuous at $\Sigma$, this latter derivative is continuous across $\Sigma$ as well.)

Gaussian normal coordinates satisfy the following well-known lemma, whose proof we include in Appendix i for the convenience of the reader.

Lemma 2. In Gaussian normal coordinates,

$$
d s^{2}=d\left(w^{n}\right)^{2}+g_{i j} d w^{i} d w^{j},
$$

where the summation on $i$ and $j$ is from 1 to $n-1$.

Note that Lemma 1 implies that the surfaces $w^{n}=$ const. are orthogonal to the coordinate directions $\partial / \partial w^{i}$, for $i=1, \ldots, n-1$.

For a smooth metric $g$, the components of the second fundamental form are given by the following lemma:

Lemma 3. In Gaussian normal coordinates,

$$
K_{i j}=-\frac{1}{2} g_{i j, n} .
$$

Proof. For every vector field $X^{i}$, we have

$$
-K_{\sigma}^{i} X^{\sigma}=\left(\nabla_{X} n\right)^{i}=n_{, \sigma}^{i} X^{\sigma}+\Gamma_{\sigma n}^{i} X^{\sigma}=\Gamma_{\sigma n}^{i} X^{\sigma},
$$

so that

$$
K_{\sigma}^{i} X^{\sigma}=-\Gamma_{\sigma n}^{i} X^{\sigma}
$$

But

$$
\Gamma_{\sigma n}^{i}=\frac{1}{2} g^{i \tau}\left\{-g_{\sigma n, \tau}+g_{\tau \sigma, n}+g_{n \tau, \sigma}\right\}=\frac{1}{2} g^{i \tau} g_{\tau \sigma, n},
$$

where we used the fact that in Gaussian normal coordinates, $g_{i n, k}=0, i=1, \ldots, n$. Thus

$$
K_{i \sigma}=-\frac{1}{2} g_{i \sigma, n}
$$

as asserted.

In the Gaussian normal coordinates $w$ associated with a given codimensionone surface $\Sigma$ and a Lipschitz continuous metric $g=g^{L} \cup g^{R}$ (where we assume as usual that $g_{L}$ and $g_{R}$ are smooth), the metric $g$ is determined on $\Sigma$, but the first derivatives of the metric suffer a jump discontinuity at $\Sigma$. Thus the second fundamental form $K$, which depends on the first derivatives of the ambient metric $g$, also suffers a jump discontinuity at $\Sigma$. In this case it follows from Lemma 2 that $K^{L}$ and $K^{R}$, the second fundamental forms on $\Sigma$ for the metrics $g^{L}$ and $g^{R}$, respectively, are given by (3.17), for $g=g^{L}, g^{R}$, respectively. Thus the following corollary of IsRaEL is immediate.

Corollary 1. The metric components of $g=g^{L} \cup g^{R}$ in Gaussian normal coordinates are $C^{1}$ functions of the coordinate variables if and only if $[K]=\left(K^{R}-K^{L}\right)=0$ at each point on the surface $\Sigma$. 
The next lemma expresses the components of the connection coefficients for the ambient metric $g$ in Gaussian normal coordinates in terms of quantities intrinsic to the shock surface. We state this for a smooth metric, and see that it applies to each side $g=g^{L}$ and $g=g^{R}$ separately when the metric is only Lipschitz continuous.

Lemma 4. The components in Gaussian normal coordinates of the connection coeffcients for a metric $g$ at a point $P \in \Sigma$ are given by

$$
\begin{gathered}
\Gamma_{i j}^{k}=\tilde{\Gamma}_{i j}^{k}, \quad i, j, k \neq n, \\
\Gamma_{i j}^{n}=K_{i j}, \quad i, j \neq n, \\
\Gamma_{i n}^{k}=-K_{i}^{k}, \quad i, k \neq n, \\
\Gamma_{i n}^{n}=0 .
\end{gathered}
$$

Here, $\tilde{\Gamma}$ denotes the $(n-1)$-dimensional connection coefficients computed from the intrinsic metric $\tilde{g}$ on $\Sigma$ with w-components $\tilde{g}_{i j}, i, j=1, \ldots, n-1$.

Proof. To obtain (3.18), use (2.4) to write

$$
\Gamma_{i j}^{k}=\frac{1}{2} g^{k \sigma}\left\{-g_{i j, \sigma}+g_{\sigma i, j}+g_{j \sigma, i}\right\} .
$$

Since $g^{k \sigma}=0$ when $\sigma=n$ and $k \neq n$, it follows that

$$
\Gamma_{i n}^{k}=\Gamma_{i n}^{\tilde{\kappa}},
$$

which is (3.18). Similarly, statement (3.19) follows from

$$
\Gamma_{i j}^{n}=\frac{1}{2} g^{n \sigma}\left\{-g_{i j, \sigma}+g_{\sigma i, j}+g_{j \sigma, i}\right\} ;
$$

statement (3.20) follows from

$$
\Gamma_{i n}^{k}=\frac{1}{2} g^{k \sigma}\left\{-g_{i n, \sigma}+g_{\sigma i, n}+g_{n \sigma, i}\right\} ;
$$

and statement (3.21) follows from

$$
\Gamma_{i n}^{n}=\frac{1}{2} g^{n \sigma}\left\{-g_{i n, \sigma}+g_{\sigma i, n}+g_{n \sigma, i}\right\}
$$

upon noting that in Gaussian normal coordinates $w$ we have $g^{n \alpha}=0$ unless $\alpha=n$, and $g_{\alpha n, \beta}=0$ for $\alpha, \beta=1, \ldots, n$.

The next lemma uses Lemmas 1 and 4 to express the components in Gaussian normal coordinates of the Riemann curvature tensor for the ambient metric $g$ in terms of quantities intrinsic to the shock surface (Gauss-Codazzi Equations). Again we state this for a smooth metric, and see that it applies to each side $g=g^{L}$ and $g=g^{R}$ separately when the metric is only Lipschitz continuous.

Lemma 5. The components in Gaussian normal coordinates of the Riemann curvature tensor for a metric $g$ at a point $P \in \Sigma$ are given by

$$
R_{j k l}^{i}=\tilde{R}_{j k l}^{i}+K_{l}^{i} K_{j k}-K_{k}^{i} K_{j l}, \quad i, j, k, l \neq n,
$$


which is equivalent to

$$
R_{k l}^{i j}=\tilde{R}_{k l}^{i j}+K_{l}^{i} K_{k}^{j}-K_{k}^{i} K_{l}^{j}, \quad i, j, k, l \neq n ;
$$

moreover,

$$
R_{i j k}^{n}=K_{i k ; j}-K_{i j ; k}, \quad i, j, k \neq n
$$

where in (3.29), the semicolon denotes covariant differentiation in the surface $\Sigma$. Statement (3.29) is equivalent to

$$
R_{j k}^{n i}=K_{k ; j}^{i}-K_{j ; k}^{i} . \quad i, j, k \neq n .
$$

Proof. For (3.27), write

$$
R_{j k l}^{i}=\Gamma_{j l, k}^{i}-\Gamma_{j k, l}^{i}+\Gamma_{\sigma k}^{i} \Gamma_{j l}^{\sigma}-\Gamma_{\sigma l}^{i} \Gamma_{j k}^{\sigma} .
$$

Thus, since only $\sigma$ can be $n$, we have

$$
R_{j k l}^{i}=\tilde{R}_{j k l}^{i}+\Gamma_{n k}^{i} \Gamma_{j l}^{n}-\Gamma_{n l}^{i} \Gamma_{j k}^{n},
$$

which by (3.18) gives (3.27). Statement (3.28) follows because $g^{i n}=0$ for $i \neq n$. For (3.29), write

$$
R_{i j k}^{n}=\Gamma_{i k, j}^{n}-\Gamma_{i j, k}^{n}+\Gamma_{\sigma j}^{n} \Gamma_{i k}^{\sigma}-\Gamma_{\sigma k}^{n} \Gamma_{i j}^{\sigma},
$$

which gives (3.29) on applying (3.19). In this case as before, (3.30) follows from (3.29) because $\tilde{g}^{i n}=g^{i n}$ when $i \neq n$.

The next lemma uses (3.28) and (3.30) to express the components in Gaussian normal coordinates of the Einstein curvature tensor for the ambient metric $g$ in terms of quantities intrinsic to the shock surface. Again we state this for a smooth metric, and see that it applies to each side $g=g^{L}$ and $g=g^{R}$ separately when the metric is only Lipschitz continuous (cf. [8]).

Lemma 6. The components in Gaussian normal coordinates of the Einstein curvature tensor for a metric $g$ at a point $P \in \Sigma$ are given by

$$
\begin{aligned}
G_{n}^{n} & \left.=\frac{1}{2}\{\operatorname{tr} K)^{2}-\operatorname{tr}\left(K^{2}\right)\right\}-\frac{1}{2} \tilde{R}, \\
G_{i}^{n} & =-\left\{(\operatorname{tr} K)_{; i}+(\operatorname{div} K)_{i}\right\},
\end{aligned}
$$

where $\tilde{R}$ denotes the curvature scalar for the metric $\tilde{g}$ intrinsic to $\Sigma$, and the semicolon denotes covariant differentiation in the surface $\Sigma$.

Proof. To prove (3.31), use (3.14) to write

$$
G_{n}^{n}=-\sum_{\sigma, \tau \neq n} R_{[\sigma \tau]}^{[\sigma \tau]}
$$

so that by (3.28)

$$
G_{n}^{n}=-\sum_{\sigma \tau \neq n} \tilde{R}_{[\sigma \tau]}^{[\sigma \tau]}+\sum_{\sigma, \tau \neq n}\left\{K_{\sigma}^{[\sigma} K_{\tau}^{\tau]}-K_{\tau}^{[\sigma} K_{\sigma}^{\tau]}\right\},
$$


where the sum must be taken over indices $\sigma<\tau$. But by definition,

$$
\widetilde{R}=\widetilde{R}_{i j}^{i j}=2 \sum_{\sigma, \tau \neq n} \widetilde{R}_{[\sigma \tau]}^{[\sigma \tau]}
$$

and

$$
(\operatorname{tr} K)^{2}-\operatorname{tr}\left(K^{2}\right)=\left(K_{i}^{i}\right)^{2}-K_{j}^{i} K_{i}^{j}=2 \sum_{i<j}\left\{K_{i}^{i} K_{j}^{j}-K_{j}^{i} K_{i}^{j}\right\} .
$$

Using these in (3.33) yields (3.31).

To prove (3.32), we use (3.15) to write

$$
-G_{i}^{n}=-\sum_{\tau \neq i, n} R_{[i \tau]}^{[n \tau]}=\sum_{j=1}^{n-1} R_{[i j]}^{n j}
$$

where we have applied the antisymmetry of the curvature tensor. Thus by (3.30),

$$
-G_{i}^{n}=\sum_{j=1}^{n-1}\left\{K_{j, i}^{j}-K_{i, j}^{j}\right\}
$$

from which (3.32) follows at once.

We can now give the

Proof of Theorem 2. Assume that $g=g^{L} \cup g^{R}$, where the metric $g$ is smooth on either side of a codimension-one shock surface $\Sigma$, and is Lipschitz continuous across the surface. Let $w$ denote the Gaussian normal coordinates associated with the surface $\Sigma$ and the metric $g$. Then we can apply (3.31) and (3.32) of Lemma 5 to $g_{L}$ from the left and $g_{R}$ from the right of $\Sigma$, respectively, to obtain

$$
\begin{aligned}
{\left[G_{n}^{n}\right] } & =\left[\frac{1}{2}\left\{(\operatorname{tr} K)^{2}-\operatorname{tr}\left(K^{2}\right)\right\}\right] \\
& =\frac{1}{2}\left\{\left(\operatorname{tr} K^{R}\right)^{2}-\operatorname{tr}\left(\left(K^{R}\right)^{2}\right)\right\}-\frac{1}{2}\left\{\left(\operatorname{tr} K^{L}\right)^{2}-\operatorname{tr}\left(\left(K^{L}\right)^{2}\right)\right\}, \\
{\left[G_{i}^{n}\right] } & =\left[\left\{-(\operatorname{tr} K)_{, i}+(\operatorname{div} K)_{i}\right\}\right] \\
& =\left\{-\left(\operatorname{tr} K^{R}\right)_{, i}+\left(\operatorname{div} K^{R}\right)_{i}\right\}-\left\{-\left(\operatorname{tr} K^{R}\right)_{, i}+\left(\operatorname{div} K^{R}\right)_{i}\right\} .
\end{aligned}
$$

Here we use the fact that $\widetilde{R}$ and $\sum_{j=1}^{n-1}\left\{K_{k i} g^{l j} \Gamma_{{ }_{l j}}^{\mathfrak{\kappa}}-K_{k j} g^{l j} \Gamma_{l i}^{\mathfrak{\kappa}}\right\}$ are equal on $\Sigma$ for $g^{L}$ and $g^{R}$ because they depend only on intrinsic properties of the metric $g$ restricted to $\Sigma$, and these agree because of the assumed continuity of $g$. But in Gaussian normal coordinates, $\boldsymbol{n}=\partial / \partial w^{n}$, and so the jump conditions (1.2) in Gaussian normal coordinates reduce to the conditions

$$
\left[G_{\alpha}^{n}\right]=0, \quad \alpha=1, \ldots, n .
$$

Now since $G$ transforms like a tensor under arbitrary $C^{1}$-coordinate transformations, the conditions (3.38) are equivalent to the statement $\left[G_{\beta}^{\alpha}\right] n_{\alpha}=0$ in the original $y$-coordinates. Thus, in light of (3.38), we conclude that (3.14) and (3.15) of Theorem 2 follow directly from (3.36) and (3.37). 
In view of Corollary 1 of Lemma 3, we can also conclude the following corollary of Theorem 2 (due to ISRAEL), which gives a global criterion for conservation across $\Sigma$ (see $[3,8])$.

Corollary 2. If $[K]=0$ at each point of $\Sigma$, then the jump conditions $\left[G_{\beta}^{\alpha}\right] n_{\alpha}=0$ must hold at the point $P$. Moreover, since in this case the metric is of class $C^{1}$ in Gaussian normal coordinates, the condition $[K]=0$ is also a necessary and sufficient condition for the original Lipschitz continuous metric components $g_{\alpha \beta}$ in the $y$ coordinates to be equivalent to a $C^{1}$ metric under a $C^{1,1}$ transformation of the coordinate variables.

Proof. The sufficiency is clear, and the necessity of this condition follows because, if the metric is equivalent to a $C^{1}$ metric under some regular $C^{1,1}$ coordinate transformation, then the mapping from these coordinates to the Gaussian normal coordinates is a $C^{2}$ mapping, and thus the metric in Gaussian normal coordinates is in $C^{1}$, which implies that the second fundamental form is continuous across the surface. (Note that $[K]=0$ at a point is not sufficient for conservation $\left[G_{j}^{i}\right] \boldsymbol{n}_{i}=0$ at the point.)

We now show that $R_{i j}$ and $G_{i j}$, viewed as second-order operators on the metric components $g_{i j}$, have delta-function singularities at a point $P \in \Sigma$ if and only if $[K] \neq 0$ at $P$. Thus, let $g=g_{L} \cup g_{R}$ be Lipschitz continuous across a shock surface $\Sigma$ in $x$-coordinates. The strategy is as follows: we first treat the case when $x$ is a Gaussian normal coordinate system defined in a neighborhood of $P \in \Sigma$. We then show that delta-function sources appear at $P \in \Sigma$ in $x$-coordinates if and only if they appear in any coordinate system related to $x$ by a $C^{1,1}$ coordinate transformation. Since any coordinate system in which $g$ is Lipschitz continuous is related to the Gaussian normal coordinates by a $C^{1,1}$ coordinate transformation, it follows that delta functions appear if and only if $[K] \neq 0$. We then show that when delta function singularities appear in $G_{i j}$ at $P \in \Sigma$ in a given coordinate system $x$, the metric is not locally Lorentzian at $P$ in the sense that there does not exist a $C^{1,1}$ coordinate transformation that takes $x$-coordinates to coordinates in which the metric is locally Lorentzian at $P$, more specifically, such that $g_{i j, k}(P)=0$. Finally, we show, surprisingly, that delta-function singularities never appear in the scalar curvature $R$ at any point on a shock wave discontinuity on either side of which $g$ is smooth, but across which $g$ is Lipschitz continuous, and this is due to a cancellation of delta functions in the sum $R_{\sigma}^{\sigma}$.

Lemma 7. Let $x$ be the Gaussian normal coordinates containing a point $P \in \Sigma$, where $\Sigma$ is any smooth surface, so that $\partial / \partial n$ is the normal direction on $\Sigma$. Then the second order n-derivatives of $g_{i j}$ that appear in the formula for the Ricci tensor $R_{i j}$ occur only in the terms $R_{i j}, i \neq n, j \neq n$, and in $R_{n n}$, and these are given by

$$
\begin{aligned}
& R_{i j}=-\frac{1}{2} g_{i j, n n}+\text { lower-order } n \text {-derivatives, } i \neq n, j \neq n, \\
& R_{n n}=\frac{1}{2} g^{\alpha \beta} g_{\alpha \beta, n n}+\text { lower-order } n \text {-derivatives, }
\end{aligned}
$$


where the sum in the last formula is taken over $\alpha, \beta \neq n$.

Proof. From (3.21), assuming Gaussian normal coordinates, we have

$$
\Gamma_{i n}^{n}=0 .
$$

Consider $R_{i j}=R_{i \sigma j}^{\sigma}$, which is given by the formulas

$$
\begin{aligned}
R_{i \beta j}^{\alpha} & =\Gamma_{i j, \beta}^{\alpha}-\Gamma_{i \beta, j}^{\alpha}+\Gamma_{\tau \beta}^{\alpha} \Gamma_{i j}^{\tau}-\Gamma_{\imath j}^{\alpha} \Gamma_{i \beta}^{\tau}, \\
R_{i j} & =R_{i \sigma j}^{\sigma}=\Gamma_{i j, \sigma}^{\sigma}-\Gamma_{i \sigma, j}^{\sigma}+\Gamma_{\tau \sigma}^{\sigma} \Gamma_{i j}^{\tau}-\Gamma_{\tau j}^{\sigma} \Gamma_{i \sigma}^{\tau} .
\end{aligned}
$$

Now since $g$ is Lipschitz continuous across $\Sigma$, and $R_{i j}$ involves second derivatives of $g$, it follows that delta functions in $R_{i j}$ can arise at $P \in \Sigma$ only in the second-order $n$-derivatives appearing in the formula for $R_{i j}$. To see this, note that in Gaussian normal coordinates, $g_{i n}=\delta_{i n}$, and $g_{i j}$ are arbitrary for $i$, $j=1, \ldots, n-1$. Thus the first derivatives in $k \neq n$ are Lipschitz continuous across $\Sigma$ because $g_{L}=g_{R}$ on $\Sigma$, and thus $g_{i j, k n}$ involves at worst jump discontinuities for $k \neq n$. Now from (3.43), the second-order $n$-derivatives can come only from $\Gamma_{i j, \sigma}^{\sigma}$ or $\Gamma_{i \sigma, j}^{\sigma}$. In the former case, this can only happen when $\sigma=n$, so consider

$$
\Gamma_{i j, n}^{n}=\frac{1}{2} g^{\sigma n}\left\{-g_{i j, \sigma n}+g_{\sigma i, j n}+g_{j \sigma, i n}\right\} .
$$

But $g^{\sigma n}=0$ unless $\sigma=n$, which implies

$$
\Gamma_{i j, n}^{n}=\frac{1}{2}\left\{-g_{i j, n n}+g_{n i, j n}+g_{j n, i n}\right\} .
$$

Thus we conclude that when $i=n$ or $j=n$, there are no non-zero second-order $n$-derivatives in $\Gamma_{i j, n}^{n}$, and when $i, j \neq n, \Gamma_{i j, \sigma}^{\sigma}$ gives rise to only one second-order $n$-derivative, namely, $\frac{1}{2} g_{i j, n n}$; i.e.,

$$
\Gamma_{i j, \sigma}^{\sigma}=\frac{1}{2} g_{i j, n n}+\text { lower-order } n \text {-derivatives. }
$$

Consider now $\Gamma_{i j, n n}^{\sigma}$, which can have second-order $n$-derivatives only for $j=n$ :

$$
\Gamma_{i \sigma, n}^{\sigma}=\frac{1}{2} g^{\sigma \tau}\left\{-g_{i \sigma, \tau n}+g_{\tau i, \sigma n}+g_{\sigma \tau, i n}\right\} .
$$

Now the first two terms $g_{i \sigma, \tau n}$ and $g_{i i, \sigma n}$ inside the bracket in (3.46) can have second-order $n$-derivatives only when $\sigma=n$ or $\tau=n$, in which case $\sigma=n=\tau$ (because $g_{i n}=0$ ), which implies that both of these terms are zero because $\Gamma_{i n}^{n}=0$. But the third term $g_{\sigma \tau, \text { in }}$ in the bracket in (3.46) has second-order $n$-derivatives only when $i=n$, and thus we have

$$
\Gamma_{n \sigma, n}^{\sigma}=\frac{1}{2} g^{\alpha \beta} g_{\alpha \beta, n n}+\text { lower-order } n \text {-derivatives, }
$$

and $\Gamma_{i \sigma, j}^{\sigma}$ is a lower-order $n$-derivative if $i \neq n$ or $j \neq n$. Thus we conclude that the second-order $n$-derivatives in the Ricci tensor occur only in the terms $R_{i j}, i \neq n$, $j \neq n$, and in $R_{n n}$, and these are given by (3.39) and (3.40).

We now consider the scalar curvature $R$ and the curvature tensors $R_{i j}$ and $G_{i j}$ as second-order distribution derivatives of the metric components $g_{i j}$ in Gaussian normal coordinates when $g$ is only Lipschitz continuous on $\Sigma$. In general we 
expect that second-order distribution derivative of $g$ introduce delta-function singularities on $\Sigma$. The following corollary gives necessary and sufficient conditions for the appearance of such delta-function singularities on $\Sigma$.

Corollary 3. Let $g=g_{L} \cup g_{R}$ be any metric that is Lipschitz continuous across a shock surface $\Sigma$, and smooth on either side of $\Sigma$. Then in Gaussian normal coordinates the scalar curvature $R$, viewed as a second-order distribution derivative of the metric components $g_{i j}$, has at worst a jump discontinuity at each $P \in \Sigma$; the Ricci and Einstein curvature tensors $R_{i j}$ and $G_{i j}$ have delta-function singularities at $P \in \Sigma$ if and only if $[K] \neq 0$ at $P$.

Proof. Assuming Gaussian normal coordinates, we have from (3.39) and (3.40) that

$$
R=g^{\sigma \tau} g_{\sigma \tau, n n}-g^{i j} g_{i j, n n}+\text { lower-order } n \text {-derivatives. }
$$

and thus the formula for $R$ in terms of $g$ contains no second-order $n$-derivatives in Gaussian normal coordinates for any Lipschitz continuous shock wave, and hence $R$ is at most discontinuous on $\Sigma$. Moreover, in Gaussian normal coordinates, $K_{i j}=g_{i j, n}, i, j \neq n$, and hence if $[K] \neq 0$ at $P \in \Sigma$, then $g_{i j, n}$ must suffer a jump discontinuity at $P$ for some $(i, j), i, j \neq n$. Thus by (3.39), $R_{i j}$ is given by the delta function $g_{i j, n n}$ plus a discontinuous function. Conversely, if $[K]=0$ at $P \in \Sigma$, then $g_{i j, n n}$ is at most discontinuous at $P$, and thus $R_{i j}$ is at most discontinuous at $P$. Since $G_{i j}=R_{i j}-\frac{1}{2} R g_{i j}$, and $R$ is at most discontinuous, we conclude that in Gaussian normal coordinates, $R_{i j}$ and $G_{i j}$ contain delta-function singularities if and only if $[K] \neq 0$.

Now let $\mathscr{R}=R_{j k l}^{i}$ denote the components of the full Riemann curvature tensor in $x$-coordinates, and let $\overline{\mathscr{R}}=\bar{R}_{\beta y \delta}^{\alpha}$ denote the components in a coordinate system $y$ related to $x$ by a $C^{1,1}$ coordinate transformation. Note that in any coordinate system, the components of the curvature are given by (3.42), and hence are determined by the same second-order differential operator $L$ on the metric components, thus $\mathscr{R}=L[g]$, and $\overline{\mathscr{R}}=L[\bar{g}]$. We note that the highest-order derivative terms in $L$ are of the form of a function of the unknowns $g_{i j}$ times linear second-order differential operators. Thus it is possible to define solutions $g$ that have only weak (distributional) derivatives of second order. The following lemma demonstrates that curvature tensors defined from $L$ in the weak sense continue to transform by the tensor transformation laws under arbitrary $C^{1,1}$ transformations of the coordinates.

Lemma 8. Let $\mathscr{R}$ be a weak solution of $\mathscr{R}=L[g]$ in $x$-coordinates. Then $\overline{\mathscr{R}}=\mathscr{R}(\partial x / \partial y)$ is a weak solution of $\overline{\mathscr{R}}=L[\bar{g}]$ for any coordinate system y related to $x$ by a $C^{1,1}$ coordinate transformation, where we use the short-hand notation

$$
\mathscr{R} \frac{\partial x}{\partial y}=\mathscr{R}^{i}{ }_{k k l} \frac{\partial x^{j}}{\partial y^{\beta}} \frac{\partial x^{k}}{\partial y^{\gamma}} \frac{\partial x^{l}}{\partial y^{\delta}} \frac{\partial y^{\alpha}}{\partial x^{i}},
$$

and multiplication by a function is taken in the weak sense. 
Proof. For smooth $g$ and smooth test functions $\varphi$, let

$$
\int_{\boldsymbol{R}^{4}} L[g] \varphi=\int_{\boldsymbol{R}^{4}} L^{*}[g, \varphi]
$$

where $L^{*}[g, \varphi]$ denotes the expression obtained from $L(g)$ by integrating the second-order derivatives in $g$ once by parts. Since the second-order derivatives in $L$ are given by ${ }^{5}$

$$
\begin{aligned}
R_{j k l}^{i}= & \Gamma_{j l, k}^{i}-\Gamma_{j k, l}^{i}+\text { 1.o.t. } \\
= & \left(g^{i \sigma}\left\{-g_{j l, \sigma}+g_{\sigma j, l}+g_{l \sigma, j}\right\}\right)_{, k} \\
& -\left(g^{i \sigma}\left\{-g_{j k, \sigma}+g_{\sigma j, l}+g_{k \sigma, j}\right\}\right)_{, l}+\text { 1.o.t., } \\
= & g^{i \sigma}\left\{-g_{j l, \sigma k}+g_{l \sigma j k}+g_{j k, \sigma l}-g_{k \sigma, j l}\right\}+\text { l.o.t., }
\end{aligned}
$$

i.e., are of the form $g^{m n} g_{i j, k l}$, it follows that $L^{*}[g, \varphi]$ contains at worst products of the metric entries $g_{i j}$, the test function $\varphi$, and their first derivatives. Thus the integral in the weak formulation $\int_{R^{4}} L^{*}[g, \varphi]$ is finite for any Lipschitz continuous metric $g$ and any Lipschitz continuous test function $\varphi$ of compact support.

Now assume that $\mathscr{R}=R_{j k l}^{i}$ is a weak solution of $\mathscr{R}=L[g]$, i.e., $\mathscr{R}$ is a linear functional on the space of Lipschitz continuous test functions (a distribution) that satisfies

$$
\langle\mathscr{R}, \varphi\rangle \equiv \int_{\boldsymbol{R}^{4}} \mathscr{R} \varphi=\int_{\boldsymbol{R}^{4}} L^{*}[g, \varphi]
$$

for every Lipschitz continuous test function $\varphi$. Note that if $\partial x / \partial y$ is Lipschitz continuous, then the derivatives are bounded, and thus if we let $\bar{g}=g(\partial x / \partial y)$ be short-hand notation for

$$
\bar{g} \equiv \bar{g}_{\alpha \beta}=g_{i j} \frac{\partial x^{i}}{\partial y^{\alpha}} \frac{\partial x^{j}}{\partial y^{\beta}} \equiv g \frac{\partial x}{\partial y},
$$

then $L^{*}[g(\partial x / \partial y), \varphi]$ is bounded for any Lipschitz continuous test function $\varphi$.

So to prove the lemma, let $g$ be an arbitrary (non-degenerate) Lipschitz continuous metric, let $\varphi$ be an arbitrary Lipschitz continuous test function, and assume that the coordinate systems $x$ and $y$ are related by a $C^{1,1}$ coordinate transformation (so that, in particular, both $\partial x / \partial y$ and $\partial y / \partial x$ are regular, Lipschitz continuous maps). Let $\bar{g}^{\varepsilon}$ denote a smooth regularization of the metric $\bar{g}_{\alpha \beta}$, and let $x^{\varepsilon}(y)$ denote a regularization of the coordinate map $x(y)$ so that $x^{\varepsilon}(y)$ is smooth and has a smooth inverse. We can clearly choose these regularizations so that $\bar{g}_{\alpha \beta}^{\varepsilon} \rightarrow \bar{g}_{\alpha \beta}$ in $C^{0,1}, \quad x^{\varepsilon}(y) \rightarrow x(y) \quad$ in $C^{1,1}, \quad \partial x^{\varepsilon}(y) / \partial y \rightarrow \partial x(y) / \partial y \quad$ in $C^{0,1}$ and $\partial y\left(x^{\varepsilon}\right) / \partial x^{\varepsilon} \rightarrow \partial y(x) / \partial x$ in $C^{0,1}$. Then

$$
\begin{gathered}
g^{\varepsilon} \equiv \bar{g}^{\varepsilon} \frac{\partial y}{\partial x^{\varepsilon}} \rightarrow g, \\
\bar{g}^{\varepsilon} \rightarrow \bar{g}
\end{gathered}
$$

\footnotetext{
${ }^{5}$ Here "l.o.t." denotes "lower-order terms", i.e., terms that contain lower order $n$-derivatives.
} 
in $C^{0,1}$. Define

$$
\begin{aligned}
\overline{\mathscr{R}}^{\varepsilon} & =L\left(\bar{g}^{\varepsilon}\right), \\
\mathscr{R}^{\varepsilon} & =\overline{\mathscr{R}}^{\varepsilon} \frac{\partial y}{\partial x^{\varepsilon}} .
\end{aligned}
$$

Now it follows directly from definitions that

$$
\left\langle\mathscr{R}^{\varepsilon} \frac{\partial y}{\partial x^{\varepsilon}}, \varphi\right\rangle=\left\langle\overline{\mathscr{R}}^{\varepsilon}, \varphi\right\rangle=\int_{\boldsymbol{R}^{4}} L^{*}\left[g^{\varepsilon} \frac{\partial y}{\partial x^{\varepsilon}}, \varphi\right] .
$$

But (3.53) simply says that $\mathscr{R}^{\varepsilon}\left(\partial y / \partial x^{\varepsilon}\right)$ is the curvature tensor for the metric $g^{\varepsilon}\left(\partial y / \partial x^{\varepsilon}\right)$, and since everything in (3.53) is smooth, we know from the fact that the curvature transforms as a tensor that $\mathscr{R}^{e}$ must be the curvature tensor for the metric $g^{\varepsilon}$; i.e., since everything in (3.53) is smooth, we know that (3.53) holds for every $\varphi \in C^{0,1}$ if and only if

$$
\left\langle\mathscr{R}^{\varepsilon}, \varphi\right\rangle=\int_{R^{4}} L^{*}\left[g^{\varepsilon}, \varphi\right]
$$

holds for every $\varphi \in C^{0,1}$. Since $g^{\varepsilon} \rightarrow g$ in $C^{0,1}$, (3.54) implies that, as $\varepsilon \rightarrow 0, \mathscr{R}^{\varepsilon}$ tends in the sense of distributions to the distribution $T$, where $T$ satisfies

$$
\langle T, \varphi\rangle=\int_{\boldsymbol{R}^{4}} L^{*}[g, \varphi] .
$$

Therefore (3.55) demonstrates that $T=\mathscr{R}$ as a distribution. Thus, in the limit $\varepsilon \rightarrow 0$, we conclude from (3.55) that $\mathscr{R}^{2} \rightarrow \mathscr{R}$, from (3.53) that $\overline{\mathscr{R}} \rightarrow \overline{\mathscr{R}}$, and hence from (3.52) that $\overline{\mathscr{R}}^{x}=\mathscr{R}(\partial y / \partial x)$ in the sense of distributions. This completes the proof of the lemma.

From this we conclude that if the Riemann curvature tensor has no deltafunction singularities at $P \in \Sigma$ in $x$-coordinates, then it has no delta-function singularities in any coordinates $y$ that are related to $x$ by a $C^{1,1}$ coordinate transformation (cf. [8]).

Theorem 3. Assume that $g=g_{L} \cup g_{R}$ is smooth on either side of a 3-dimensional shock surface $\Sigma$, and is Lipschitz continuous across $\Sigma$. Then the scalar curvature $R$, when viewed as a second-order operator (in the weak sense) on the metric components $g_{i j}$, produces at most a jump discontinuity (i.e., no delta-function singularities) at $P \in \Sigma$, and the curvature tensors $R_{j k l}^{i}, R_{i j}$ and $G_{i j}$ produce no delta-function singularities at $P \in \Sigma$ if and only if the jump in the second fundamental form $K$ satisfies $[K]=0$ at $P$.

Proof. By Corollary 1, the theorem is true in Gaussian normal coordinates $x$, and thus by Corollary 3 and Lemma 8 it holds in any coordinate system $y$ which is $C^{1,1}$ related to $x$. Since for any metric $g=g_{L} \cup g_{R}$ which is smooth on either side of $\Sigma$ and Lipschitz continuous across $\Sigma$, the transformation to Gaussian normal coordinates is an invertible $C^{1,1}$ coordinate transformation, the theorem follows at once. 
As a direct corollary of Theorem 3 we see that there exists a locally Lorentzian coordinate frame in a neighborhood of a point $P$ on a Lipschitz continuous shock surface $\Sigma$ if and only if $[K]=0$ at $P$; namely, we have

Corollary 4. Assume that $g=g_{L} \cup g_{R}$ is smooth on either side of a 3-dimensional shock surface $\Sigma$, and is Lipschitz continuous across $\Sigma$ in a coordinate system $y$ defined in a neighborhood of $P \in \Sigma$. Then there exists a regular $C^{1,1}$ coordinate transformation $y \rightarrow x$ such that $x$ is locally Lorentzian for $g$ at $P\left(g_{i j}=\eta_{i j}\right.$ and $g_{i j, k}=0$ at $\left.P\right)$ if and only if $[K]=0$ at $P$.

Proof. Assume that $[K]=0$ at $P$, and choose locally Lorentzian coordinates at $P$ for the smooth metric obtained by restricting $g$ to the surface $\Sigma$ in a neighborhood of $P$ in the surface $\Sigma$. Extend these coordinates to Gaussian normal coordinates $x$ based on these surface coordinates, the $x$ coordinates being defined in an $n$-dimensional neighborhood of $P$. Then in $x$ coordinates the metric components $g_{i j}$ satisfy $g_{i j}=\eta_{i j}$ and $K_{i j}=g_{i j, n}=0$ at $P$, and so $x$ is locally Lorentzian at $P$. Conversely, assume that $[K] \neq 0$, but that there exists a coordinate transformation $y \rightarrow x$ such that, in $x$-coordinates, $g_{i j}=\eta_{i j}$ and $g_{i j, k}=0$ at $P$. Then in $x$-coordinates, $g$ is of class $C^{1}$ at $P$, and hence there are no delta-function singularities in the components $g_{i j}$ of $g$ in $x$-coordinates. Thus by Theorem 3, $[K]=0$, and hence the locally Lorentzian coordinates $x$ cannot exist when $[K] \neq 0$.

The following corollary directly implies Theorem 1 of the Introduction:

Corollary 5. Assume that the components of $g=g_{L} \cup g_{R}$ in a coordinate system $y$ are Lipschitz continuous across a smooth 3-dimensional shock surface $\Sigma$ and are $C^{k}$ functions of $y$ on either side of $\Sigma$, and that all $k$ derivatives are continuous up to the boundary $\Sigma$ from either side of $\Sigma$. Assume also that $g$ is a weak solution of $R_{\alpha \beta}=0$ or $G_{\alpha \beta}=0$ when viewed as second-order operators on the metric components $g_{\alpha \beta}$. Then in Gaussian normal coordinates $x$ (which are $C^{1,1}$ related to the original coordinates), the metric components $g_{i j}$ are actually $C^{k}$ functions of $x$ across $\Sigma$.

Proof. Assume first that $g=g_{L} \cup g_{R}$ is a weak solution of $R_{\alpha \beta}=0$. But $R_{i j}=0$ in the weak sense across $\Sigma$ implies that there are no delta-function sources in $R_{i j}$ on $\Sigma$, and thus by the previous theorem, $[K]=0$ across $\Sigma$. Thus IsRAEL's result implies that the $g_{i j, k}$ are all continuous across $\Sigma$, and since $G_{i j} \equiv 0$, the jump conditions are automatically satisfied across $\Sigma$. It follows from (3.39) and (3.40) that in Gaussian normal coordinates,

$$
\begin{aligned}
& R_{i j}=-\frac{1}{2} g_{i j, n n}+\text { lower-order } n \text {-derivatives, } i \neq n, j \neq n \\
& R_{n n}=\frac{1}{2} g^{i j} g_{i j, n n}+\text { lower-order } n \text {-derivatives. }
\end{aligned}
$$

But since the $g_{i j, k}$ are continuous across $\Sigma$, it follows that the lower-order terms in (3.56) and (3.57) must be continuous functions across $\Sigma$, our assumptions implying that the derivatives of $g$ in the surface $\Sigma$ are the same for $g_{L}$ and $g_{R}$. But since 
$R_{i j}=0$ for both $g_{L}$ and $g_{R}$, we can solve for $g_{i j, n n}$ in (3.56) and (3.57) in terms of lower-order derivatives that are continuous across $\Sigma$, and conclude that $g_{i j, n n}$ must also be continuous across $\Sigma$ for all $i, j=1, \ldots, n$. (Recall that $g_{n i}=$ constant in Gaussian normal coordinates.) This shows us that $k$ th order derivatives of $g_{i j}$ which are up to second order in $x^{n}$ are in fact continuous functions of $x$ across $\Sigma$ in Gaussian normal coordinates. Now differentiate (3.56) and (3.57) with respect to $x^{n}$. Then the differentiated lower-order terms in (3.56) and (3.57) are continuous across $\Sigma$, and hence again we can solve for $g_{i j, n n}$ in terms of functions that are continuous across $\Sigma$. Thus we conclude that $k$ th order derivatives of $g_{i j}$ which are up to second order in $x^{n}$ are in fact continuous functions of $x$ across $\Sigma$ in Gaussian normal coordinates $x$. Continuing, we see that all the $k$ th order derivatives of $g_{i j}$ are continuous across $\Sigma$ in Gaussian normal coordinates. Since, by Corollary 3 , the scalar curvature never contains delta-function singularities on $\Sigma$, the result for $R_{i j}$ implies the same result for $G_{i j}$.

The same argument establishes the following more general version of this corollary:

Corollary 6. Assume that $g=g_{L} \cup g_{R}$ is smooth on either side of a 3-dimensional shock surface $\Sigma$, and is Lipschitz continuous across $\Sigma$ in some coordinate system $y$. Assume that $g$ is a weak solution of $G_{\alpha \beta}=\kappa T_{\alpha \beta}$ that contains no delta-function singularities on $\Sigma$. Then in Gaussian normal coordinates the metric components $g_{i j}$ are $C^{2}$ functions of $x$ if and only if $[G]=0$ across $\Sigma$.

Summary. The results of this section are summarized in the following theorem:

Theorem 4. Let $\Sigma$ denote a smooth, 3-dimensional shock surface in space-time with spacelike normal vector $\boldsymbol{n}$. Assume that the components $g_{i j}$ of the gravitational metric $g$ are smooth on either side of $\Sigma$ (continuous up to the boundary on either side separately) and Lipschitz continuous across $\Sigma$ in some fixed coordinate system. Then the following statements are equivalent:

(i) $[K]=0$ at each point of $\Sigma$.

(ii) The curvature tensors $R_{j k l}^{i}$ and $G_{i j}$, viewed as second-order operators on the metric components $g_{i j}$, produce no delta-function sources on $\Sigma$.

(iii) For each point $P \in \Sigma$ there exists a $C^{1,1}$ coordinate transformation defined in a neighborhood of $P$, such that, in the new coordinates (which can be taken to be the Gaussian normal coordinates for the surface) the metric components are $C^{1,1}$ functions of these coordinates.

(iv) For each $P \in \Sigma$, there exits a coordinate frame that is locally Lorentzian at $P$, and can be reached within the class of $C^{1,1}$ coordinate transformations.

Moreover, if any one of these equivalencies hold, then the Rankine-Hugoniot jump conditions $[G]_{i}^{\sigma} n_{\sigma}=0$ (which express the weak form of conservation of energy and momentum across $\Sigma$ when $G=\kappa T$ ) hold at each point on $\Sigma$.

Here $[K]$ denotes the jump in the second fundamental form (extrinsic curvature) $K$ across $\Sigma$ (this being determined by the metric separately on each side of 
$\Sigma$ because $\mathrm{g}_{i j}$ is only Lipschitz continuous across $\Sigma$ ). By $C^{1,1}$ we mean that the first derivatives are Lipschitz continuous. Theorem 1 should be credited mostly to IsRAEL, who obtained results (i) (iii) in Gaussian normal coordinates. Our contribution is to identify the covariance class of $C^{1,1}$ transformations, and thereby to obtain precise coordinate-independent statements for (ii) and (iii), as well as to show the equivalence with (iv). As a consequence of this, we obtain the result that the Ricci scalar curvature $R$ never has delta-function sources at a Lipschitz continuous matching of the metrics, as well as the results in Corollaries 5 and 6 which validate the statement that shock-wave singularities in the source-free Einstein equations $R_{i j}=0$ or $G_{i j}=0$ can only appear as coordinate anomalies, and can be transformed away by coordinate transformation. Note that when there are delta-function sources in $G$ on a surface $\Sigma$, the surface should be interpreted as a surface layer (because $G=\kappa T$ ), and not a true shock wave [3, 8]. In Lemma 9, Section 5, below, we show for spherically symmetric solutions that $[G]_{i}^{\sigma} n_{\sigma}=0$ alone implies the absence of surface layers (and hence the other equivalencies in Theorem 5), so long as the areas of the spheres of symmetry match smoothly at $\Sigma$. We use this result in our construction of the shock waves that extend the Oppenheimer-Snyder model to the case of non-zero pressure. The following counter-example shows that in general, the above equivalencies can fail even when $\left[G_{i}^{\sigma}\right] n_{\sigma}=0$ holds at each point on $\Sigma{ }^{6}$

For the counter-example it suffices to show that there exist Lipschitz continuous shock waves which satisfy the Israel jump relations (3.3) and (3.4) across a shock-wave interface, but which cannot be transformed to a metric that is in class $C^{1}$ in a neighborhood of each point on the shock. By Corollary 1, it suffices to construct a shock-wave interface across which the Israel conditions are satisfied, but such that the second fundamental form $K$ is not continuous across the interface. To this end, let $g_{i j}$ denote the coordinates of a metric in Gaussian normal coordinates, such that the spacelike normal to the shock surface is given by $\boldsymbol{n}=\partial / \partial x^{n}$, and $g_{i j}$ is of the form

$$
g_{i j}=\left[\begin{array}{cc}
h_{i j} & 0 \\
0 & 1
\end{array}\right]
$$

Assume now that the $h_{i j}$ are given by

$$
h_{i j}= \begin{cases}\eta_{i j}+a_{i j} x^{n} & \text { if } x^{n}>0 \\ \eta_{i j}+b_{i j} x^{n} & \text { if } x^{n}<0\end{cases}
$$

where $a_{i j}$ and $b_{i j}$ are constants to be determined. Thus by Lemma 3, the second fundamental forms $K^{L}$ and $K^{R}$ on the left and right of the shock surface are given by $K_{i j}^{L}=a_{i j}$ and $K_{i j}^{R}=b_{i j}, i, j=1, \ldots, n-1$. Since $K_{i j}^{L}$ and $K_{i j}^{R}$ are constant,

$$
K_{i, \sigma}^{\sigma}=(\operatorname{tr} K)_{, i}=0,
$$

\footnotetext{
${ }^{6}$ See [3] where such an example is given in which $G \equiv 0$ on both sides of $\Sigma$.
} 
for $K=K^{L}, K^{R}$. Thus the Israel jump conditions (3.3) and (3.4) reduce to

$$
\left[(\operatorname{tr} K)^{2}-\operatorname{tr}\left(K^{2}\right)\right]=0 .
$$

Hence to satisfy the Israel jump conditions it suffices to find $\boldsymbol{a} \equiv a_{i j}$ and $\boldsymbol{b} \equiv b_{i j}$ satisfying

$$
(\operatorname{tr} a)^{2}-\operatorname{tr}\left(a^{2}\right)=0=(\operatorname{tr} b)^{2}-\operatorname{tr}\left(b^{2}\right) .
$$

But in the simplest case where $\boldsymbol{a}$ and $\boldsymbol{b}$ are $2 \times 2$ matrices,

$$
\begin{aligned}
\operatorname{tr} a & =a_{11}+a_{22}, \\
\operatorname{tr}\left(\boldsymbol{a}^{2}\right) & =a_{11}^{2}+2 a_{21} a_{12}+a_{22}^{2},
\end{aligned}
$$

and so

$$
(\operatorname{tr} \boldsymbol{a})^{2}-\operatorname{tr}\left(\boldsymbol{a}^{2}\right)=2 \operatorname{det} \boldsymbol{a} .
$$

Thus we can satisfy the Israel jump conditions by choosing $\boldsymbol{a}$ and $\boldsymbol{b}$ to be any $2 \times 2$ matrices with zero determinant. If in addition, $a_{i j} \neq b_{i j}$, then $[K]=K^{R}-K^{L} \neq 0$, and so by Theorem 2, the conservation $\left[G_{i}^{n}\right]=0$ holds across the interface $x^{n}=0$, but, in view of Corollary 1 , the metric cannot be transformed to a metric that is globally of class $C^{1}$ across the shock.

\section{A Generalization of the Oppenheimer-Snyder Model}

In this section we construct a generalization of the Oppenheimer-Snyder model for gravitational collapse in spherically symmetric solutions of the Einstein equations (1.1). It is well known that the Oppenheimer-Snyder model requires the simplifying assumption that the pressure $p$ be identically zero. We shall construct a corresponding solution when the pressure is given by an arbitrary barotropic equation of state $p=p(\rho)$, and in this section we allow the equation of state to be chosen separately on either side of the shock surface.

The Oppenheimer-Snyder model is a spherically symmetric solution of the Einstein equations that is constructed by matching the (empty-space) Schwarzschild solution to the Robertson-Walker (R-W) solution (a spherically symmetric homogeneous solution) across an interface which is interpreted as the boundary of a collapsing star. The R-W metric is a solution with $T$ given by (2.9), and the Schwarzchild solution has stress tensor $T \equiv 0$, which implies that, if energy and momentum are conserved, no energy or momentum can cross any interface that connects the two solutions. This is the case in the Oppenheimer-Snyder model where the pressure is taken to be identically zero. We generalize this to the case $p \neq 0$ by matching the $\mathrm{R}-\mathrm{W}$ metric to the Oppenheimer-Tolman (O-T) metric [14], a spherically symmetric solution of the Einstein equations with nonzero stressenergy tensor of the form (2.9). In the limit $p=0$, the shock interface goes over to a contact discontinuity [11], and we recover the Oppenheimer-Snyder solution $[6,7,9,10]$. 
In the case of non-zero pressure, energy and momentum must cross the interface between the two solutions, and thus the interface propagates like a gas-dynamical shock wave. When $p \neq 0$, conservation of energy and momentum does not hold automatically across the shock interface; this problem will be considered in the next section. Here we show that the R-W metric and the $\mathrm{O}-\mathrm{T}$ metric can be matched in a Lipschitz continuous manner for arbitrary equations of state on both sides of the shock. In addition, we identify a global principle of conservation of mass that applies to these matched solutions. In the following section, in order to force the additional constraint of conservation to hold across the shock interface, we allow the pressure on one side to be determined dynamically with the shock position. We end the next section with the derivation of an autonomous systems of two ordinary differential equations that determine the $\mathrm{O}-\mathrm{T}$ shock waves across which conservation holds. When conservation fails to hold, the surface is a boundary layer and carries mass and momentum (see [3]).

We begin by introducing three metrics which are spherically symmetric solutions of the Einstein equations relevant to our development: the Schwarzschild, the Oppenheimer-Tolman (O-T) and the Robertson-Walker (R-W) metric. These are defined, respectively, by (cf. [15, Chapter 11]):

$$
\begin{gathered}
d \bar{s}^{2}=-\left(1-\frac{2 \mathscr{G} M}{\bar{r}}\right) d \bar{t}^{2}+\left(1-\frac{2 \mathscr{G} M}{\bar{r}}\right)^{-1} d \bar{r}^{2}+\bar{r}^{2} d \Omega^{2} \\
M \equiv \text { Const. }
\end{gathered}
$$

$(\mathrm{O}-\mathrm{T})$

$$
d \bar{s}^{2}=-B(\bar{r}) d \bar{t}^{2}+A(\bar{r})^{-1} d \bar{r}^{2}+\bar{r}^{2} d \Omega^{2},
$$

$$
d s^{2}=-d t^{2}+R^{2}(t)\left\{\frac{1}{1-k r^{2}} d r^{2}+r^{2} d \Omega^{2}\right\}
$$

(The first two metrics are written in barred coordinates so that they can be distinguished from the unbarred coordinates when we do the matching of metrics below.) The quantity $d \Omega^{2}=d \theta^{2}+\sin ^{2} \theta d \varphi^{2}$ denotes the standard metric on the 2-sphere. The metric (4.1) describes an empty-space solution of the Einstein equations (1.1) generated by a fixed total mass-energy $M$ (which, hereafter in this section, we refer to as the mass) centered at the origin. The metric in (4.2) describes the interior of a star, and satisfies (1.1) with stress-energy tensor given by that of a perfect fluid (2.9):

$$
T_{i j}=\bar{p} g_{i j}+(\bar{p}+\bar{\rho}) u_{i} u_{j}
$$

where in this section we assume a barotropic equation of state $\bar{p}=\bar{p}(\bar{\rho})$, and we take $c=1$. Here, $A$ is given by

$$
A(\bar{r})=\left(1-\frac{2 \mathscr{G} M}{\bar{r}}\right)
$$


where $M \equiv M(\bar{r})$ and $\bar{\rho} \equiv \bar{\rho}(\bar{r})$ satisfy the following system of ordinary differential equations:

$$
\begin{gathered}
\frac{d M}{d \bar{r}}=4 \pi \bar{r}^{2} \bar{\rho}, \\
-\bar{r}^{2} \frac{d}{d \bar{r}} \bar{p}=\mathscr{G} M \bar{\rho}\left\{1+\frac{\bar{p}}{\bar{\rho}}\right\}\left\{1+\frac{4 \pi \bar{r}^{3} \bar{p}}{M}\right\}\left\{1-\frac{2 \mathscr{G} M}{\bar{r}}\right\}^{-1} .
\end{gathered}
$$

The equation (4.7) is referred to by WEINBERG as the fundamental equation of Newtonian astrophysics, with general-relativistic corrections supplied by the last three factors [15, page 301$]$. The metric component $B \equiv B(\bar{r})$ in (4.2) satisfies the equation

$$
\frac{B^{\prime}(\bar{r})}{B}=-2 \frac{\bar{p}^{\prime}(\bar{r})}{\bar{p}+\bar{\rho}} .
$$

Notice that the equations (4.6) and (4.7) are equations that determine $M(\bar{r})$ and $\bar{\rho}(\bar{r})$ once we specify the equation of state $\bar{p}=\bar{p}(\bar{\rho})$. The total mass is then given by

$$
M(\bar{r})=\int_{0}^{\bar{r}} 4 \pi \xi^{2} \bar{\rho}(\xi) d \xi .
$$

The coordinates $(t, r, \theta, \varphi)$ in (4.2) are assumed to be "co-moving" with respect to the source fluid. That is, coordinates are said to be co-moving relative to a background diagonal metric $g_{i j}$ if the spatial components of the 4-velocity $u$ of the fluid vanish, $u^{i}=0, i=1,2,3$. Since $u$ is a unit vector relative to $g$, i.e., since

$$
g^{i j} u_{i} u_{j}=-1,
$$

it follows that for diagonal metrics,

$$
u_{0}=\sqrt{-g_{00}} .
$$

In case of the I-S metric (4.2), equation (4.11) becomes

$$
u_{t}=\sqrt{B(\bar{r})}
$$

Finally, for the R-W metric (4.3), which describes a spherically symmetric, homogeneous space-time, the field equations (1.1) imply that the function $R(t)$ (the "cosmological scale factor", cf. [15]), satisfies the following equations (see [15, Chapters 11 and 14]):

$$
\begin{gathered}
3 \ddot{R}=-4 \pi \mathscr{G}(\rho+3 p) R, \\
R \ddot{R}+2 \dot{R}^{2}+2 k=4 \pi \mathscr{G}(\rho-p) R^{2},
\end{gathered}
$$

together with

$$
\dot{p} R^{3}=\frac{d}{d t}\left\{R^{3}(p+\rho)\right\} .
$$


Equation (4.15) is equivalent to

$$
\frac{d}{d R}\left(\rho R^{3}\right)=-3 p R^{2}
$$

Substituting (4.13) into (4.14) we get

$$
\dot{R}^{2}+k=\frac{8 \pi \mathscr{G}}{3} \rho R^{2} .
$$

In the R-W metric (4.3), $\rho$ and $p$ are assumed to be functions of $t$ alone, in which case equations (4.16) and (4.17) give two equations for the two unknowns $R$ and $\rho$, again for an equation of state of the form $p=p(\rho){ }^{7}$ The coordinates for the R-W metric in (4.3) are also assumed to be co-moving with respect to the fluid, and in this case (4.11) implies that

$$
u_{r}=u_{\theta}=u_{\varphi}=0, \quad u_{t}=1 .
$$

Note that if $R(t)$ and $\rho(t)$ satisfy (4.13)-(4.15), then so does $R(-t)$ and $\rho(-t)$. Moreover, from (4.15), we find

$$
\dot{p} R^{3}=R^{3}(\dot{p}+\dot{\rho})+3 R^{2} \dot{R}(p+\rho) .
$$

This implies that

$$
R \dot{\rho}+3 \dot{R}(p+\rho)=0
$$

from which we conclude that

$$
\dot{\rho} \dot{R}<0 .
$$

Thus to every expanding solution there exists a corresponding contracting solution, and conversely.

We now construct a coordinate system $(\bar{t}, \bar{r})$ for the R-W metric and a shock surface written in these coordinates so that the metrics (4.2) and (4.3) match in a Lipschitz continuous manner along the surface in the $(\bar{t}, \bar{r})$-coordinates. To do this we must define a coordinate mapping that takes the unbarred frame of the R-W metric (4.3) over to a barred coordinate system that leaves fixed the $\theta$ and $\varphi$ coordinates and is consistent with the coordinates in the O-T solution (4.2). To ensure that the areas of the 2-spheres agree in the two frames, we require

$$
\bar{r}^{2} d \Omega^{2}=R^{2} r^{2} d \Omega^{2},
$$

and thus choose

$$
r=R r^{8}
$$

\footnotetext{
${ }^{7}$ Note that $p$ and $\bar{p}$ need not satisfy the same equation of state.

${ }^{8}$ Note that at this stage the transformation $\bar{r}=R r$ is defined globally, and in the development below it is important that this equality hold in an open neighborhood of the shock surface in order to ensure conservation, (cf. Lemma 9, equation (5.3)).
} 
In order to rewrite the R-W metric in $(t, \bar{r})$-coordinates, we have from (4.20) that

$$
d \bar{r}=R d r+\dot{R} r d t
$$

so

$$
d r=\frac{1}{R} d \ddot{r}-\frac{\dot{R}}{R} r d t
$$

and thus

$$
d r^{2}=\frac{1}{R^{2}} d \bar{r}^{2}+\frac{\dot{R}^{2}}{R^{2}} r^{2} d t^{2}-2 \frac{\dot{R}}{R^{2}} \bar{r} d t d \bar{r}
$$

Thus, the R-W metric (4.3) is given in $(t, \bar{r})$-coordinates by

$$
d s^{2}=-\left\{1-\frac{\dot{R}^{2} \bar{r}^{2}}{R^{2}-k \bar{r}^{2}}\right\} d t^{2}+\frac{R^{2}}{R^{2}-k \bar{r}^{2}} d \bar{r}^{2}-\frac{2 R \dot{R} \vec{r}}{R^{2}-k \bar{r}^{2}} d t d \vec{r}+\bar{r}^{2} d \Omega^{2},
$$

which, by using

$$
\left\{R^{2}-k \bar{r}^{2}-\dot{R}^{2} \bar{r}^{2}\right\}=R^{2}\left\{1-\frac{8 \pi \mathscr{G}}{3} \rho R^{2} r^{2}\right\}
$$

becomes

$$
d s^{2}=\frac{1}{R^{2}-k \bar{r}^{2}}\left\{-R^{2}\left(1-\frac{8 \pi \mathscr{G}}{3} \rho R^{2} r^{2}\right) d t^{2}+R^{2} d \bar{r}^{2}-2 R \dot{R} \bar{r} d t d \bar{r}\right\}+\bar{r}^{2} d \Omega^{2} .
$$

We next define a mapping $t=t(\vec{t}, \vec{r})$ that eliminates the cross term $d t d \bar{r}$ in (4.25). We do this first for a general metric of the form

$$
d \tilde{s}^{2}=-C(t, \bar{r}) d t^{2}+D(t, \bar{r}) d \bar{r}^{2}+2 E(t, \bar{r}) d t d \bar{r} .
$$

It is not hard to verify that if $\psi=\psi(t, \vec{r})$ is chosen to satisfy the equation

$$
\frac{\partial}{\partial \bar{r}}(\psi C)=-\frac{\partial}{\partial t}(\psi E)
$$

then

$$
d \bar{t}=\psi(t, \bar{r})\{C(t, \bar{r}) d t-E(t, \bar{r}) d \bar{r}\},
$$

is an exact differential. With this choice, the $(\bar{t}, \bar{r})$ line element for (4.26) becomes

$$
d \tilde{s}^{2}=-\left(\psi^{-2} C^{-1}\right) d \bar{t}^{2}+\left(D+\frac{E^{2}}{C}\right) d \bar{r}^{2} .
$$

Now in terms of the metric

$$
d \tilde{s}^{2}=-R^{2}\left[1-\frac{8 \pi \mathscr{G}}{3} \rho R^{2} r^{2}\right] d t^{2}+R^{2} d \bar{r}^{2}-2 R \dot{R} \bar{r} d \bar{t} d \bar{r}
$$


which appears in (4.25), $C, D$ and $E$ are given by

$$
\begin{aligned}
& C=R^{2}\left\{1-\frac{8 \pi \mathscr{G}}{3} \rho R^{2} r^{2}\right\}, \\
& D=R^{2}, \\
& E=-R \dot{R} \bar{r} .
\end{aligned}
$$

Thus, in view of $(4.30)$, the R-W metric in $(\bar{t}, \bar{r})$-coordinates becomes

$$
d s^{2}=\frac{1}{R^{2}-k \bar{r}^{2}}\left\{-\left(\psi^{2} C\right)^{-1} d \bar{t}^{2}+\left(D+\frac{E^{2}}{C}\right) d \bar{r}^{2}\right\}+\bar{r}^{2} d \Omega^{2} .
$$

But from (4.31)-(4.33) we obtain

$$
D+\frac{E^{2}}{C}=R^{2}+\frac{R^{2} \dot{R} \bar{r}^{2}}{R^{2}\left(1-\frac{8 \pi}{3} \mathscr{G} \rho \bar{r}^{2}\right)}=R^{2}+\frac{\dot{R} R^{2} r^{2}}{1-\frac{8 \pi}{3} \mathscr{G} \rho R^{2} r^{2}} .
$$

Now equating the $d \bar{r}^{2}$ coefficients in the O-T solution (4.2) and the R-W solution (4.34) and using (4.35), we obtain the equation for the shock surface: ${ }^{9}$

$$
\left(R^{2}-k \bar{r}^{2}\right)\left(1-\frac{2 \mathscr{G} M}{\bar{r}}\right)^{-1}=R^{2}+\frac{\dot{R}^{2} R^{2} r^{2}}{1-\frac{8 \pi}{3} \mathscr{G} \rho R^{2} r^{2}},
$$

which (4.17) simplifies to

$$
M(\bar{r})=\frac{4 \pi}{3} \rho(t) \bar{r}^{3} .
$$

Hence (4.37) defines the shock surface, and the shock surface in $(t, r)$-coordinates can be obtained from (4.37) by making the substitution $\bar{r}=R(t) r$. (We assume that the shock surface remains within the domain of definition of the R-W metric, namely, that $1-k r^{2}>0$, when $k>0$.) It remains only to determine $\psi$ from (4.27) so that the $d \bar{t}^{2}$ terms in the O-T and R-W metric agree on this surface. To obtain $\psi$, which globally determines the coordinate $t$ in terms of the $(\bar{t}, \bar{r})$ coordinates for the R-W metric, we solve (4.27) subject to initial data on the shock surface which are forced upon us by the condition that the $d \bar{t}^{2}$ terms match on the shock surface. That is,

$$
\frac{1}{R^{2}-k \bar{r}^{2}} \frac{1}{\psi^{2} C}=B(\bar{r})
$$

on the shock surface (4.37). To carry out this program, we rewrite (4.27) in the form of a first-order linear partial differential equation for $\psi$,

$$
C \psi_{\bar{r}}+E \psi_{t}=f(t, \bar{r}, \psi)
$$

Here, $C$ and $E$ are functions of $t$ and $\bar{r}$ given by (4.31) and (4.33), and thus we can solve the initial-value problem (4.39) in $(t, \bar{r})$-coordinates with initial data (4.38)

\footnotetext{
${ }^{9}$ Note that, interestingly, the $d \bar{r}^{2}$ coefficients are independent of $\psi$.
} 
given on the shock surface (4.37), provided that the shock surface is non-characteristic for (4.39).

Now the characteristics for (4.39) are given by

$$
\lambda \equiv \frac{d \bar{r}}{d t}=\frac{C}{E},
$$

so that the function $\psi$ is obtained by solving the ordinary differential equation

$$
\frac{d \psi}{d \mu}=f(t, \bar{r}, \psi),
$$

starting with initial values on the shock surface (4.37), where $d / d \mu$ denotes differentiation in the $(E, C)$-direction in $(t, \bar{r})$-coordinates. Solving (4.38) for $\psi$ gives the initial values of $\psi$ to be met on the shock surface, namely,

$$
\psi^{2}=\frac{1}{B\left(R^{2}-k \dot{r}^{2}\right) C} .
$$

Thus, if $d \bar{r} / d t$ denotes the speed of the shock surface in $(t, \bar{r})$-coordinates, then by (4.40) the condition that the shock surface be non-characteristic at a point is that

$$
\frac{d \bar{r}}{d t} \neq \frac{C}{E}
$$

If (4.43) holds at a point on the shock surface (4.37), then we can solve (4.39) uniquely for $\psi$ in a neighborhood of the point, thereby matching the R-W and O-T solutions in a Lipschitz continuous manner in a neighborhood of such a point on the surface in the $(\bar{t}, \bar{r})$-coordinate system. Since we need only define local coordinate systems in order to define a space-time manifold, the shock surface (4.37) defines a complete Lipschitz matching of the metrics R-W and O-T at each point of the surface where the non-characteristic condition (4.43) holds. It is interesting to observe that one need not explicitly solve the partial differential equation (4.39) for $\psi$ in order to determine the shock-surface equation (4.37), and the solution of (4.37) can be calculated even when the $\bar{t}$-coordinate, defined in terms of $\psi$, cannot be constructed. This has obvious numerical implications.

We consider the condition (4.43) below in Propositions 2 and 3, but first we discuss the equation for the shock surface (4.37). This is necessary in order to obtain an expression for the shock speed, and to motivate the conditions in Propositions 2 and 3 below. We begin by noting that we have not made any choice regarding whether the R-W metric is on the "inside" or the "outside" of the O-T solution. For the case of a star, the R-W metric is on the inside (at small values of $\bar{r}$ within the shock surface) and the $\mathrm{O}-\mathrm{T}$ is on the outside of the shock surface. For definiteness, we only consider this case, although the discussion we give below applies equally well to the case when the R-W metric is on the outside. Note first that (4.37) allows an interpretation of a global principle of conservation of mass in the special coordinate $\bar{r}$. Indeed, $M\left(\bar{r}_{0}\right)$ is the total mass that would appear inside the radius $\bar{r}_{0}$ were the Oppenheimer-Tolman solution continued to values of $\bar{r}<\bar{r}_{0}$. Thus, $M(\bar{r})$ represents the total mass that is generating the $\mathrm{O}-\mathrm{T}$ solution outside the radius 
$\bar{r}=\bar{r}_{0}$. This describes the left-hand side of (4.37). The right-hand side of (4.37) can be interpreted as the total mass inside the sphere of radius $\bar{r}_{0}$ at a fixed time $t$ in the Robertson-Walker solution. This says that the "total mass" is conserved as the shock propagates, if we compute the total mass in $\bar{r}$-coordinates at fixed $t$ for $\bar{r}<\bar{r}_{0}$, and at fixed $\bar{t}$ for $\bar{r}>\bar{r}_{0}$. Therefore, we have shown that the total mass in the $\mathrm{O}-\mathrm{T}$ solution that an observer sees out at infinity is fixed, and this equals the total mass in the inside R-W metric plus the total mass in the outside $\mathrm{O}-\mathrm{T}$ metric, so long as these latter masses are computed from the densities in the $\bar{r}$-coordinate at fixed $t$ and $\bar{t}$, respectively. As an application of this global principle of conservation of mass, we note that since in a "physically relevant" model for a star, the density $\bar{\rho}(\bar{r})$ for the O-T metric should be decreasing function of $\bar{r}$, the global conservation principle cannot hold when $\bar{\rho}-\rho \equiv[\rho]=0$ across the shock surface. Indeed, if $d \bar{\rho} / d \bar{r}<0$ for $\bar{r}<\bar{r}_{0}$, and $\rho\left(t_{0}\right)=\bar{\rho}\left(\bar{r}_{0}\right)$, then

$$
\frac{4 \pi}{3} \rho\left(t_{0}\right) \bar{r}^{3}=\frac{4 \pi}{3} \bar{\rho}\left(\bar{r}_{0}\right) \bar{r}^{3}<\int_{0}^{\bar{r}_{0}} 4 \pi \bar{\rho}(\xi) \xi^{2} d \xi=M\left(\bar{r}_{0}\right),
$$

and so by (4.37), the point $\left(t_{0}, \bar{r}_{0}\right)$ cannot lie on the shock surface. That is, the global conservation of mass principle implies that if $d \bar{\rho} / d \bar{r}<0$, then $[\rho] \neq 0$ across the shock.

With this motivation, we can now calculate the shock speed unless the condition $[\rho] \neq 0$. Indeed, by the implicit function theorem, the shock surface (4.37) is given by $\bar{r}=\bar{r}(t)$ provided that

$$
\frac{d M}{d \ddot{r}}-4 \pi \rho(t) \ddot{r}^{2} \neq 0 .
$$

But, using (4.6) enables us to write (4.45) as

$$
4 \pi \bar{r}^{2}(\bar{\rho}-\rho) \neq 0,
$$

at a point on the shock surface. Thus, as we have shown above, if we assume that $d \bar{\rho} / d \bar{r}<0$, this condition is always valid on the shock surface. We can now calculate the speed of the shock $s \equiv \dot{\bar{r}}$, (where "dot" denotes $d / d t$ ). Using (4.37), which we write in the form

$$
M(\bar{r}(t))=\frac{4 \pi}{3} \rho(t) \bar{r}(t)^{3},
$$

and differentiating with respect to $t$, we find

$$
s \equiv \dot{\bar{r}}=\frac{\dot{\rho} \bar{r}}{3[\rho]} .
$$

Since $[\rho]<0$ (we are assuming that $d \bar{\rho} / \partial \bar{r}<0$ ), the shock speed is negative if $\dot{\rho}>0$ and is positive if $\dot{\rho}<0$. Observe that, from (4.40), the condition on the shock speed (4.48) that guarantees that the surface be non-characteristic at a point is (cf. (4.42))

$$
\left(\frac{\dot{\rho} \bar{r}}{3[\rho]}\right)^{2} \neq \frac{C^{2}}{E^{2}}=\frac{\left(\frac{8 \pi}{3} \mathscr{G} \rho \bar{r}^{2}-1\right)^{2}}{\frac{8 \pi}{3} \mathscr{G} \rho \bar{r}^{2}-k r^{2}},
$$


where we have used (4.17), (4.20), (4.31), (4.33). Note that in the classical theory of shock waves, only the shock waves that move into the fluid with lower pressure are stable, and the corresponding shock waves that move into the higher-pressure side are unstable, and are referred to as rarefaction shocks [11]. This means that if $d \bar{\rho} / \bar{r}>0$, then the shock is stable if $s>0(\dot{\rho}<0)$, and unstable if $s<0(\dot{\rho}>0)$. We remark that all of the above development is independent of the equations of state $p=p(\rho)$ and $\bar{p}=\bar{p}(\bar{\rho})$. In the limiting case of OPPENHEIMER \& SNYDeR [9], the pressure $p \equiv 0$, and the O-T solution is replaced by the Schwarzschild solution (4.1) having a constant mass function $M(\bar{r}) \equiv M=$ const. In this case the R-W solution satisfies $\rho(t) R(t)^{3}=\rho(0)$, and so for a particular solution satisfying $R(0)=1$, $\dot{R}(0)=0,(4.17)$ implies that $k=\frac{8}{3} \pi^{\mathscr{G}}$. Thus (4.37) gives the well-known result that the radius of the star $a$ at time $t=0$ is given by the relation (see [15, page 346])

$$
M=\frac{4 \pi}{3} \rho(0) a^{3} .
$$

The following proposition is useful.

Proposition 1. On the shock surface (4.37) the following identities hold:

$$
\begin{aligned}
\frac{1}{\psi^{2} C^{2}} & =B\left(1+\frac{A E^{2}}{C^{2}}\right)=\frac{B}{A}\left(1-k r^{2}\right), \\
C & =R^{2} A, \\
\frac{E}{C} & =\frac{-\dot{R} r}{A} \\
\frac{E^{2}}{C^{2}} & =\frac{-A+\left(1-k r^{2}\right)}{A^{2}}, \\
\dot{R}^{2} r^{2} & =-A+\left(1-k r^{2}\right) .
\end{aligned}
$$

The proof is given in Appendix ii.

We end this section by giving conditions under which the shock surface is non-characteristic; i.e., that (4.42) holds. We assume here that the shock surface lies within the domain of definition of the R-W metric if $k>0$. The first proposition gives conditions on the equation of state $\bar{p}(\bar{\rho})$ that guarantee that the shock surface (4.37) is non-characteristic provided it does not intersect the Schwarzschild radius $A=1-(2 \mathscr{G} M / \bar{r})=0$ of the O-T solution.

Proposition 2. If the equation of state $\bar{p}(\bar{\rho})$ satisfies the condition

$$
\frac{d \bar{p}}{d \bar{\rho}} \geqq 0,
$$

and if

$$
A \neq 0
$$

everywhere on the shock surface (4.37), then the shock surface is nowhere characteristic. 
Proof. We already have that (cf. (4.40), (4.33))

$$
\begin{aligned}
& \lambda=\frac{C}{E}=\frac{C}{R(-\dot{R}) \bar{r}}, \\
& s=\frac{\dot{\rho} \bar{r}}{3[\rho]} .
\end{aligned}
$$

From the equation (4.7) for $d \bar{p} / d \bar{r}$, we see that the sign of $d \bar{p} / d \bar{r}$ is positive inside the Schwarzschild radius and negative outside. Thus $\operatorname{sign}([\rho])=\operatorname{sign}$ $(d \bar{p} / d \bar{r})=-\operatorname{sign}(A)$. But on the shock surface, we also have by (4.51) that

$$
C=R^{2} A,
$$

and so $\operatorname{sign}(A)=\operatorname{sign}(C)$. Finally, we also have from (4.19) that $\dot{R} \dot{\rho}<0$. Thus,

$$
\begin{aligned}
\operatorname{sign}(\lambda)-\operatorname{sign}(s) & =\operatorname{sign}(C)-\operatorname{sign}([\rho]) \\
& =-\left\{\operatorname{sign}\left(\frac{d \vec{p}}{d \bar{r}}+\operatorname{sign}([\rho])\right)\right\} \neq 0 .
\end{aligned}
$$

We shall also need the following proposition:

Proposition 3. If $\dot{R}=0$ and $A \neq 0$ at a point on the shock surface (4.37) (i.e., the point is not on the Schwarzschild radius), then, if the shock speed is finite at the point, the shock surface is also non-characteristic at the point.

Proof. By (4.39), the characteristic surfaces satisfy

$$
\begin{aligned}
& \frac{d \bar{r}}{d s}=C=R^{2} A, \\
& \frac{d t}{d s}=E=-R^{3} \dot{R},
\end{aligned}
$$

where we have used (4.51) and (4.52). Therefore, if $\dot{R}=0$, the characteristic is tangent to $t=$ constant, and thus any finite speed $s=d \bar{r} / d t$ is a non-characteristic speed.

Summary. The results of this section can be summarized as follows: Let (4.2) and (4.3) denote the O-T and R-W metrics for arbitrarily chosen equations of state $\bar{p}=\bar{p}(\bar{\rho})$ and $p=p(\rho)$, respectively. Then we have identified the following conditions under which there exists a smooth regular coordinate transformation

$$
\Psi:(t, r) \rightarrow(\bar{t}, \bar{r}),
$$

and a corresponding shock surface $r=r(t)$ in $(t, r)$-coordinates (which maps to the curve $\bar{r}=\bar{r}(\bar{t})$ in barred coordinates by $(\bar{t}, \bar{r}(\bar{t}))=\Psi(t, r(t)))$, such that the metrics (4.2) and (4.3) agree and are Lipschitz continuous across the shock surface when written in the same coordinates (for example, they agree when both are written in either the barred or unbarred coordinates); namely, we have the following theorem: 
Theorem 5. Assume that the shock surface $\bar{r}=\bar{r}(t)$ is defined implicitly by

$$
M(\bar{r})=\frac{4 \pi}{3} \rho \bar{r}^{3}
$$

in a neighborhood of a point $\left(\bar{t}_{0}, \bar{r}_{0}\right)$ that satisfies (4.58). Assume that

$$
\Psi_{2}(t, r)=R(t) r=\bar{r},
$$

so that the spheres of symmetry agree in the barred and unbarred frames, and the shock surface in $(t, r)$-coordinates is given by $r(t)=\bar{r}(t) / R(t)$. Assume finally that both

$$
\begin{gathered}
1-k r(t)^{2}>0, \\
\frac{d \bar{r}}{d t} \neq \frac{C}{E}=-\frac{\dot{R} r}{A}
\end{gathered}
$$

hold at $t=t_{0}$ (cf. (4.31), (4.33) and (4.52)). Then the coordinate $\bar{t}=\Psi_{1}(t, r)$ can be defined smoothly and in such a way that $\Psi=\left(\Psi_{1}, \Psi_{2}\right)$ is one-to-one and regular in a neighborhood of the point $\left(t_{0}, r_{0}\right),(c f .(4.39))$, and the metrics (4.2) and (4.3) match in a Lipschitz continuous fashion across the shock surface $r=r(t)$ in a neighborhood of $\left(t_{0}, r_{0}\right)$.

Note that by the implicit function theorem, a sufficient condition for (4.58) to define a surface locally through $\left(t_{0}, \bar{r}_{0}\right)$ is that

$$
\frac{\partial}{\partial \bar{r}} \frac{M}{\bar{r}^{3}}=M-\frac{4 \pi}{3} \bar{\rho} \neq 0 .
$$

By differentiating (4.58) directly, we obtain the alternative sufficient condition (cf. (4.46))

$$
[\rho] \neq 0 .
$$

\section{An Extension of the Oppenheimer-Snyder Model Satisfying Conservation}

In the last section we constructed a generalization of the Oppenheimer-Snyder model to the case of non-zero pressure by matching the R-W metric to the $\mathrm{O}-\mathrm{T}$ metric in a Lipschitz continuous fashion across the shock surface (4.37). Two important problems remain to be answered: first, what is the smoothness class of the matched metrics, and second, under what conditions does conservation of energy and momentum hold across the shock interface? In this section we answer these questions by deriving the constraint equations on the $\mathrm{R}-\mathrm{W}$ metric that guarantees conservation to hold across the shock surface when an arbitrary O-T solution is given. We show that conservation imposes one (not two!) additional constraints, and so we can meet this constraint by allowing the R-W pressure to be an additional unknown. We then reduce the constraint of conservation to an autonomous system of ordinary differential equations in which the coefficients are defined implicitly through identities obtained in the previous section. In a 
forthcoming paper we shall prove the existence of local solutions and the positivity of the R-W pressure for these equations.

The following lemma asserts that the smoothness problem and the conservation problem are related:

Lemma 9. Assume that $g$ and $\bar{g}$ are two spherically symmetric metrices that match Lipschitz continuously across a three-dimensional shock interface $\Sigma$ to form the matched metric $g \cup \bar{g}$. That is, assume that $g$ and $\bar{g}$ are Lorentzian metrics given by

$$
\begin{aligned}
& d s^{2}=-a(t, r) d t^{2}+b(t, r) d r^{2}+c(t, r) d \Omega^{2}, \\
& d \bar{s}^{2}=-\bar{a}(\bar{t}, \bar{r}) d \bar{t}^{2}+\bar{b}(\bar{t}, \bar{r}) d \bar{r}^{2}+\bar{c}(\bar{t}, \bar{r}) d \Omega^{2},
\end{aligned}
$$

and that there exists a smooth coordinate transformation $\Psi:(t, r) \rightarrow(\bar{t}, \bar{r})$, defined in a neighborhood of a shock surface $\Sigma$ given by $r=r(t)$, such that the metrics agree on $\Sigma$. (We implicitly assume that $\theta$ and $\varphi$ are continuous across the surface.) Assume that

$$
c(t, r)=\bar{c}(\Psi(t, r))
$$

in an open neighborhood of the shock surface $\Sigma$, so that, in particular, the areas of the 2-spheres of symmetry in the barred and unbarred metrics agree on the shock surface. Assume also that the shock surface $r=r(t)$ in unbarred coordinates is mapped to the surface $\bar{r}=\bar{r}(\bar{t})$ by $(\bar{t}, \bar{r}(\bar{t}))=\Psi(t, r,(t))$. Assume, finally, that the normal $\boldsymbol{n}$ to $\Sigma$ is non-null, and that $\boldsymbol{n}(c) \neq 0$ where $\boldsymbol{n}(c)$ denotes the derivative of the function $c$ in the direction of the vector $n .{ }^{10}$ Then the following are equivalent to the statement that the components of the metric $g \cup \bar{g}$ in any Gaussian normal coordinate system are $C^{1,1}$ functions of these coordinates across the surface $\Sigma$ :

$$
\begin{aligned}
{\left[G_{j}^{i}\right] n_{i} } & =0, \\
{\left[G^{i j}\right] n_{i} n_{j} } & =0, \\
{[K] } & =0 .
\end{aligned}
$$

Here $[f]=\bar{f}-f$ denotes the jump in the quantity $f$ across $\Sigma$, and $K$ is the second fundamental form on the shock interface defined by (2.10). ${ }^{11}$

Proof. Let $\left(w^{1}, w^{2}, w^{3}\right)=\left(z^{1}, \theta, \varphi\right)$ be a smooth coordinate system on $\Sigma$, and let $z=\left(z^{0}, \ldots, z^{3}\right)$ denote the extension of these coordinates to a Gaussian normal

${ }^{10}$ I.e., we assume that the areas of the 2 -spheres of symmetry change monotonically in the direction normal to the surface. E.g., if $c=r^{2}$, then $\partial c / \partial t=0$, so the assumption $n(c) \neq 0$ is valid except when $\boldsymbol{n}=\partial / \partial t$, in which case the rays of the shock surface would be spacelike. Thus the shock speed would be faster than the speed of light rays if our assumption $\boldsymbol{n}(c) \neq 0$ failed in the case $c=r^{2}$.

11 This does not contradict the spherical shell example of ISRAEL in [3] because (5.3) fails in that example. 
coordinate system in a neighborhood of $\Sigma$. Here, in the case of space-time, we let $\partial / \partial z^{n}=\partial / \partial z^{0}$ (cf. Appendix i). Then by Lemma 2 of Section 3, $\boldsymbol{n}=\partial / \partial z^{3}$, and $\boldsymbol{T}=\partial / \partial z^{1}$ is tangent to the shock surface. Now in light of Corollary 2 of Theorem 2 it suffices to verify that (5.5) implies (5.6). By Theorem 2, in w-coordinates we have

$$
\left[G^{i j}\right] n_{i} n_{j}=\left[G^{00}\right]=\left[\operatorname{tr}\left(K^{2}\right)-(\operatorname{tr} K)^{2}\right]
$$

But in Gaussian normal coordinates the metric $g \cup \bar{g}$ is diagonal on the surface $\Sigma$. To see this, note that the restriction of the metric $(g \cup \bar{g})$ to the surface $\Sigma$ is diagonal because the off-diagonal $\varphi$ and $\theta$ components are zero in both (5.1) and (5.2), and the metric components $(g \cup \bar{g})_{0 j}$ for $j \neq 0$ are zero throughout any Gaussian normal coordinate frame in a whole neighborhood of $\Sigma$. Thus, by Lemma 3 of Section 3,

$$
K_{i j}=-\frac{1}{2} g_{i j, 0}
$$

Therefore, since $g \cup \bar{g}$ is diagonal on $\Sigma, K$ is also diagonal, and so the only non-zero components of $K$ are

$$
\begin{aligned}
& K_{11}=-\frac{1}{2} g_{11,0}, \\
& K_{22}=-\frac{1}{2} g_{22,0}, \\
& K_{33}=-\frac{1}{2} g_{33,0} .
\end{aligned}
$$

But, since $c$ and $\bar{c}$ (defined in (5.1) and (5.2)) transform like functions under arbitrary $(t, r)$-transformations, (5.3) implies that $c$ and $\bar{c}$ define the same invariant function in a neighborhood of $\Sigma$. Thus, by (5.3) and the fact that $c=g_{22}=\bar{c}=\bar{g}_{22}$ on $\Sigma$, we see that $g_{22,0}=\boldsymbol{n}(c)=\bar{g}_{22,0} \neq 0$ and $g_{33,0}=\boldsymbol{n}(c) \sin ^{2} \theta=\bar{g}_{33,0} \neq 0$ on the surface $\Sigma$, and hence

$$
\begin{aligned}
& {\left[K_{22}\right]=0,} \\
& {\left[K_{33}\right]=0}
\end{aligned}
$$

across $\Sigma$. Now we have

$$
\left.0=\left[G_{i j}\right] n_{i} n_{j}=\left[G_{00}\right]=\left[\operatorname{tr}\left(K^{2}\right)-\operatorname{tr} K\right)^{2}\right]=-2\left[K_{11}\right]\left(K_{22}+K_{33}\right),
$$

and since $\left(K_{22}+K_{33}\right) \neq 0$ (by the assumption $\left.n(c) \neq 0\right)$, we conclude that (5.5) and (5.14) imply that

$$
\left[K_{11}\right]=0 .
$$

Since $K_{i j}$ is diagonal, (5.12), (5.13) and (5.15) imply (5.6), so (5.5) implies (5.6), and we are done.

Now in order to obtain the constraint equations for conservation of energy and momentum, we begin by assuming that a known O-T solution is given; namely, we 
assume that $\bar{\rho}(\bar{r}), \bar{p}(\bar{r}), B(\bar{r}) A(\bar{r})$ and $M(\bar{r})$ are known functions that satisfy (cf. $(4.5)-(4.8))$

$$
\begin{aligned}
d \bar{s}^{2} & =-B d \bar{t}^{2}+A^{-1} d \bar{r}^{2}+\bar{r}^{2} d \Omega^{2}, \\
A\left(\bar{r}^{2}\right) & =1-\frac{2 \mathscr{G} M(\bar{r})}{\bar{r}}, \\
-\bar{r}^{2} \frac{d \bar{p}}{d \bar{r}} & =\mathscr{G} M \bar{\rho} A^{-1}\left[1+\frac{\bar{p}}{\bar{\rho}}\right]\left[1+\frac{4 \pi \bar{r}^{3} \bar{p}}{M}\right], \\
\frac{d M}{d \bar{r}} & =4 \pi \bar{r}^{2} \bar{\rho}, \\
\frac{B^{\prime}(\bar{r})}{B} & =-\frac{2 \bar{p}^{\prime}(\bar{r})}{\bar{p}+\bar{\rho}} .
\end{aligned}
$$

Note that when the equation of state $\bar{p}=\bar{p}(\bar{\rho})$ is specified, (4.6) and (4.7) yield a system of equations that determine $\bar{\rho}$ and $M$. Now assuming the barred O-T metric is given, we attempt to find $R(t)$ and $k$ such that the $\mathrm{R}-\mathrm{W}$ metric

$$
d s^{2}=-d t^{2}+R^{2}\left\{\frac{d r^{2}}{1-k r^{2}}+r^{2} d \Omega^{2}\right\}
$$

matches the metric (5.16) across the shock surface defined implicitly by

$$
M(\bar{r})=\frac{4 \pi}{3} \rho(t) \bar{r}^{3},
$$

and such that conservation holds across the surface. To do this we proceed formally. Assume first that the hypotheses (4.58)-(4.61) of Theorem 3, Section 4, hold, so that the metrics match Lipschitz continuously across the surface (5.2). In particular, we assume for our derivation of the conservation constraints that the shock surface can be written as

$$
\rho=\frac{3}{4 \pi} \frac{M(\bar{r})}{\bar{r}^{3}}=f(\bar{r})
$$

that $f^{\prime}(\bar{r}) \neq 0$, so that the shock surface defines $\bar{r}$ as a function of $\rho$ via

$$
\bar{r}=f^{-1}(\rho)=\bar{r}(\rho),
$$

and that the identities (4.50)-(4.54) of Proposition 1 hold all along the shock surface. Assuming this and assuming that the shock surface is given by $r=r(t)$, we derive a system of ordinary differential equations in $(R, r)$ that express conservation across the surface. Note that since $R r=\bar{r}$ holds in a neighborhood of the shock surface, (5.3) of Lemma 9 holds. In this section, we finish the paper by obtaining the differential equations for a shock surface across which conservation holds, under the assumptions of Lemma 9. We investigate the regime in which such shocks are physical in a forthcoming paper. To start, use the Einstein equation $G=\kappa T$, the condition $\left[G^{i j}\right] n_{i} n_{j}=0$ for conservation across the shock (cf. Lemma 7), and the 
assumption that the source fluid is co-moving with respect to the metrics on either side of the shock (cf. (4.4)) to rewrite the condition for conservation as

$$
[T]^{i j} n_{i} n_{j}=(p-\bar{p})|n|^{2}+(\rho+p) n_{0}^{2}-(\bar{\rho}+\bar{p}) \frac{\bar{n}_{0}^{2}}{B}=0 .
$$

Here we let $n_{i}$ and $\bar{n}_{i}$ denote the $i$-coordinates of the normal vector $\boldsymbol{n}$ to the shock surface (5.22) in unbarred (R-W) and barred (O-T) coordinates, respectively, and $|\boldsymbol{n}|^{2}=g^{i j} n_{i} n_{j}$. (Note that $u^{i}=\delta_{0}^{i}$ in R-W coordinates, $u^{i}=B^{-1 / 2} \delta_{0}^{i}$ in O-T coordinates, thus giving rise to the factor $B$.) Since $n_{i}=0=\bar{n}_{i}, i=2,3$, we need only pay attention to the 0 - and 1 -coordinates of $\boldsymbol{n}$. To verify (5.25), note that, for example, in the R-W unbarred frame (4.4) gives

$$
T^{i j} n_{i} n_{j}=p g^{i j} n_{i} n_{j}+(p+\rho)\left(u^{i} n_{i}\right)^{2}=p|\boldsymbol{n}|^{2}+(p+\rho)\left(n_{0}\right)^{2} .
$$

Moreover, we need not choose the vector $\boldsymbol{n}$ to be of unit length, so long as $n_{i}$ and $\bar{n}_{i}$ are the coordinates of the same vector. Since the left-hand side of (5.25) is an invariant scalar, so is the right-hand side. In order to evaluate $n_{i}$ and $\bar{n}_{i}$, let (5.22) (formally) define the surface $r=r(t)$, which we can write as the level curve of the scalar $\varphi(t, r)=r-r(t)=0$. Then we can choose $n_{i} d x^{i}=d \varphi$, so that

$$
d \varphi=n_{0} d t+n_{1} d r=-\dot{r} d t+d r
$$

which yields

$$
\begin{aligned}
& n_{0}=-\dot{r}, \\
& n_{1}=1 .
\end{aligned}
$$

To obtain $\bar{n}_{i}$, we write the function $\varphi$ in $(\bar{t}, \bar{r})$-coordinates:

$$
\varphi(\bar{t}, \bar{r})=\frac{\bar{r}}{R(t(\bar{t}, \bar{r}))}-r(t(\bar{t}, \bar{r}))
$$

Then

$$
d \varphi(\bar{t}, \bar{r})=\left\{-\frac{\bar{r}}{R^{2}} \dot{R} \frac{\partial t}{\partial \bar{t}}-\dot{r} \frac{\partial t}{\partial \bar{t}}\right\} d \bar{t}+\bar{n}_{1} d \bar{r}=-\frac{\dot{\bar{r}}}{R} \frac{\partial t}{\partial \bar{t}} d \bar{t}+\bar{n}_{1} d \bar{r}
$$

so that

$$
\bar{n}_{0}=-\frac{\dot{\bar{r}}}{R} \frac{\partial t}{\partial \bar{t}}
$$

But using the fact that

$$
\bar{r}=R r
$$

together with (4.28):

$$
d \bar{t}=\psi(t, \bar{r})\{C(t, \bar{r}) d t-E(t, \bar{r}) d \bar{r}\},
$$

we have

$$
d t=(\psi C)^{-1} d \bar{t}+\frac{E}{C} d \bar{r}
$$


which implies that

$$
\frac{\partial t}{\partial t}=(\psi C)^{-1}
$$

Putting this into (5.30) yields

$$
\bar{n}_{0}=-\frac{\dot{\bar{r}}}{R \psi C} .
$$

Using the identity (4.50) of Proposition 1 we obtain

$$
\bar{n}_{0}^{2}=\frac{B}{A}\left(1-k r^{2}\right) \bar{r}^{2},
$$

where $-B$ and $A^{-1}=\left(1-\frac{2 \mathscr{G} M}{\bar{r}}\right)^{-1}$ are the coefficients of $d \bar{t}^{2}$ and $d \bar{r}^{2}$ in the O-T metric (4.2). Finally, using the R-W metric (5.21) to compute $|\boldsymbol{n}|^{2}$, we obtain

$$
|\boldsymbol{n}|^{2}=-n_{0}^{2}+\frac{1-k r^{2}}{R^{2}} n_{1}^{2}=-\dot{r}^{2}+\frac{1-k r^{2}}{R^{2}} .
$$

Now substituting (5.26), (5.32) and (5.33) into (5.25) yields

$$
[T]^{i j} n_{i} n_{j}=(\bar{p}+\rho) \dot{r}^{2}-(\bar{\rho}+\bar{p}) \frac{1-k r^{2}}{A R^{2}} \dot{r}^{2}+(p-\bar{p}) \frac{1-k r^{2}}{R^{2}}=0 .
$$

Equation (5.34) gives the additional constraint imposed by conservation across the shock in terms of the quantities $r=r(t)$ (the shock position) and the values that $\rho, p, \bar{\rho}, \bar{p}$ and $R$ take on the shock surface. The following proposition explains why the pressure must be taken to be zero in the Oppenheimer-Snyder model:

Lemma 10. If $\bar{\rho}=\bar{p}=0$ identically (so that the $O$ - $T$ solution reduces to the Schwarzschild solution (4.1)), and $p \geqq 0$ and $p \geqq 0$ everywhere, then (5.34) implies that $p=0$ and $r(t)=$ const. all along the shock.

Proof. When $\bar{\rho}=\bar{p}=0,(5.34)$ reduces to

$$
\rho \dot{r}^{2}+p \frac{1-k r^{2}}{R^{2}}=0
$$

Since $\left(1-k r^{2}\right) / R^{2}>0$ in the R-W metric, the lemma follows at once.

We now derive an ordinary differential equation for the unknowns $(r(t)$, $R(t)$ ), the shock position $r(t)$ and the cosmological scale factor $R(t)$ in the R-W metric, so that we can guarantee that solutions of the ordinary differential equation necessarily give the shock-wave interfaces that are conservative. In order that the functions $R, \rho$ and $p$ in the R-W metric not be over-constrained when we impose the conservation constraint (5.34), we must allow the pressure $p$ in the R-W metric to be determined by the unknown functions $r$ and $R$, rather than by an 
equation of state $p=p(\rho)$. To see this, recall that the $\mathrm{R}-\mathrm{W}$ metric satisfies the equations, (cf. [15])

$$
\begin{aligned}
\dot{R}^{2} & =\frac{8 \pi \mathscr{G}}{3} \rho R^{2}-k \\
p & =-\frac{\frac{d}{d t}\left(\rho R^{3}\right)}{3 R^{2} \dot{R}}
\end{aligned}
$$

In the case when $p$ is given by equation of state $p=p(\rho)$, equations (5.35) and (5.36) give a system of two equations in two unknowns which determine $R$ and $\rho$. Thus if we also ask that (5.34) hold on the shock surface for some fixed known functions $\bar{\rho}$ and $\bar{p}$, then we obtain a third constraint on the functions $R, \rho$ and $p$, and then we let $p$ be one of the unknowns in order to have as many unknowns as equations. To construct the ordinary differential equation, note that $\bar{\rho}(\bar{r})$ and $\bar{p}(\bar{r})$ can be taken to be known functions of $r(t)$ and $R(t)$ in (5.34) through the identity

$$
\bar{r}(t)=R(t) r(t)
$$

which holds all along the shock surface. Thus, on the shock surface,

$$
\begin{aligned}
& \bar{\rho}=\bar{\rho}(R(t) r(t)), \\
& \bar{p}=\bar{p}(R(t) r(t)) .
\end{aligned}
$$

Moreover, the function $\rho$ in equations (5.34) and (5.35) can be written as a function of $(r(t), R(t))$ through the shock surface equation (5.22):

$$
\rho=f(R(t) r(t))
$$

where

$$
f(\bar{r})=\frac{3}{4 \pi} \frac{M(\bar{r})}{\bar{r}^{3}}
$$

Therefore, we can write equations (5.34) and (5.35) in terms of the two unknowns $(r, R)$ once we obtain an expression for the pressure $p$ on the shock surface in terms of $r(t)$ and $R(t)$. For this we use the final equation (5.36). Differentiating, we obtain

$$
p=-\frac{\frac{d}{d t}\left(\rho R^{3}\right)}{3 R^{2} \dot{R}}=-\rho-\frac{\dot{\rho} R}{3 \dot{R}},
$$

and using the relations

$$
\begin{aligned}
\dot{\rho} & =f^{\prime}(\bar{r})\{\dot{R} r+\dot{r} R\}, \\
f^{\prime}(\bar{r}) & =\frac{3}{4 \pi}\left(\frac{M^{\prime}}{\bar{r}^{3}}-\frac{3 M}{\bar{r}^{4}}\right)=\frac{3}{\bar{r}}(\bar{\rho}-\rho),
\end{aligned}
$$

we obtain, after simplification (cf. (5.39), (5.43)) that

$$
p=-\frac{1}{4 \pi} \frac{M^{\prime}(\bar{r})}{\bar{r}^{2}}-\frac{R^{2} f^{\prime}(\bar{r})}{3 \dot{R}} \dot{r}=-\bar{\rho}-\frac{R^{2} f^{\prime}}{3 \dot{R}} \dot{r}=-\bar{\rho}+\frac{R}{r}(\rho-\bar{\rho}) \frac{\dot{r}}{\dot{R}},
$$


which gives the final variable $p$ in terms of the unknowns $(r(t), R(t))$ and their derivatives on the shock surface. Substituting (5.44) into (5.34) yields

$$
\begin{aligned}
{[T]^{i j} n_{i} n_{j}=} & (\bar{p}+\rho) \dot{r}^{2}-(\bar{\rho}+\bar{p}) \frac{\left(1-k r^{2}\right)}{A R^{2}}(\dot{R} r+R \dot{r})^{2} \\
& +\left\{-\bar{\rho}+(\rho-\bar{\rho}) \frac{R}{r \dot{R}} \dot{r}\right\} \frac{\left(1-k r^{2}\right)}{R^{2}}-\bar{p} \frac{\left(1-k r^{2}\right)}{R^{2}}=0,
\end{aligned}
$$

which, upon multiplying by $\left(1-k r^{2}\right)^{-1}$ and simplifying, puts the constraint imposed by conservation into the final form

$$
[T]^{i j} n_{i} n_{j}=\alpha \dot{r}^{2}+\beta \dot{r}+\gamma=0,
$$

where

$$
\begin{aligned}
& \alpha=\frac{\bar{p}+\rho}{1-k r^{2}}-\frac{\bar{\rho}+\bar{p}}{A}, \\
& \beta=-2 \frac{\dot{R} r}{A R}(\bar{\rho}+\bar{p})+\frac{1}{\bar{r} \dot{R}}(\rho-\bar{\rho}), \\
& \gamma=-\left(1+\frac{\dot{R}^{2} r^{2}}{A}\right)\left(\frac{\bar{p}+\bar{\rho}}{R^{2}}\right) .
\end{aligned}
$$

For a given O-T solution $\bar{\rho}(\bar{r}), \bar{p}(\bar{r}), M(\bar{r}), A(\bar{r})=\left(1-\frac{2 \mathscr{G} M}{\bar{r}}\right), B(\bar{r})$, all of the functions appearing in (5.46) can be expressed in terms of the unknowns $(r(t), R(t))$ by means of the transformation $\bar{r}=r R$, the shock surface equation $\rho(t)=f(\bar{r}) \equiv \frac{3}{4 \pi} \frac{M}{\bar{r}^{3}}$, and the R-W identity $\dot{R}^{2}=\frac{8 \pi \mathscr{G}}{3} \rho R^{2}-k$. Thus equation (5.46) gives the conservation constraint in terms of a first-order ordinary differential equation with independent variable $t$ and unknowns $(r, R)$. It follows that the system (5.35), (5.46) forms a first-order autonomous system of two equations in the two unknowns $(r, R)$. Moreover, the solutions $(r(t), R(t))$ of this ordinary differential equation determine the R-W metrics that match a given O-T metric across a shock surface $r=r(t)$ such that the additional condition of conservation is maintained across the shock. The density and the pressure in the R-W metric are then determined by $R(t)$ through the relations $\rho(t)=f(\bar{r})$ and (5.44), respectively. We summarize this result in the following theorem:

Theorem 6. Let $M(\bar{r}), \bar{\rho}(\bar{r}), \bar{p}(\bar{r})$ be determined by a fixed $O-T$ solution of the Einstein equations, and assume the coordinate identification $\bar{r}=r R$ and $\bar{t}=\bar{t}(t, r)$, where $\bar{t}$ satisfies (4.28). Then the $R-W$ metrics $\rho(t), p(t), R(t)$ that match the given $O-T$ solution across the shock surface $r=r(t)$, such that the weak form of conservation $[T]^{i j} n_{j}=0$ holds across the surface, satisfy the system of ordinary differential equations

$$
\begin{gathered}
\dot{R}^{2}=\frac{8 \pi G}{3} \rho R^{2}-k, \\
\alpha \dot{r}^{2}+\beta \dot{r}+\gamma=0,
\end{gathered}
$$


where $\alpha, \beta, \gamma$ are the functions of $(r, R)$ given by (5.47)-(5.49). Solutions of (5.50), (5.51) then determine the $R-W$ density and pressure through the identities

$$
\begin{aligned}
\rho(t) & =\frac{3}{4 \pi} \frac{M}{\bar{r}^{3}}, \\
p & =-\frac{\frac{d}{d t}\left(\rho R^{3}\right)}{3 R^{2} \dot{R}} .
\end{aligned}
$$

Moreover (by Lemma 9), the surface $r=r(t)$, so obtained, is a true shock surface, and not a surface layer, because all of the equivalencies (i)-(iv) of Theorem 4 must hold at the surface $r=r(t)$.

The following corollary asserts that for every "collapsing" $(\dot{\rho}>0)$ solution of (5.35), (5.46) there exists a corresponding "expanding" $(\dot{\rho}<0)$ solution and vice versa.

Corollary 7. If $(r(t), R(t))$ satisfies (5.35) and (5.46), then so does $(r(-t), R(-t))$.

Proof. When $t \rightarrow-t$, the only term in (5.35) and (5.46) that changes sign is $\dot{R} \rightarrow-\dot{R}$, and thus $\beta$ changes sign in (5.46), but not $\alpha$ or $\gamma$. The result follows.

\section{Concluding Remarks}

Theorem 6 reduces the problem of constructing shock-wave solutions of the $\mathrm{O}-\mathrm{T}$ and R-W metrics to the level that the O-T and R-W metrics themselves are given; i.e., to a system of two ordinary differential equations in two unknowns. In a forth-coming publication we shall study solutions of the equations $(5.50)$ and (5.51) for the shock position $r(t)$ and the cosmological scale factor $R(t)$ which determine the pressure jump across the shock in these models. It remains to compare the qualitative behavior of solutions in the case $p \neq 0$ to the case $p=0$, the Oppenheimer-Snyder case, where there is available an explicit formula for the solution. This analysis should have implications relevant to the long-standing open problem as to whether the pressure forces can prevent "continued gravitational contraction" in stars [9].

It is also interesting to consider the consequences of admitting solutions of the Einstein equations which are only Lipschitz continuous across shock interfaces; it is not known whether such Lipschitz continuous shock waves can evolve out of smooth solutions of the field equations. Consider, for example, the case of the free Einstein equations $G_{i j}=0$. By allowing any solution metric that is only Lipschitz continuous across an interface, it appears that one could construct many solutions of $G_{i j}=0$ across the interface by solving the initial-value problem with arbitrary derivatives on the surface. (Of course this is not an issue for $C^{1}$ metrics.) In the case of spherically symmetric solutions, Birkhoff's Theorem (see [8]) tells us that shock-wave discontinuities cannot form because all solutions are coordinateequivalent to the Schwarzschild solution. This is consistent with our Lemma 7 
which states that any Lipschitz continuous spherically symmetric solution of $G_{i j}=0$ is coordinate-equivalent to a $C^{\infty}$ metric. However, our counter-example at the end of Section 3 shows that solutions having less symmetry can admit conservative shock-wave solutions $\left(\left[G_{j}^{i}\right] n_{i}=0\right)$ across which $K$ is not continuous, and hence across which the metric suffers jumps in the first derivatives which cannot be transformed away. We conclude that the introduction of Lipschitz continuous solution metrics raises interesting new questions of existence, uniqueness and admissibility for weak solutions of the Einstein equations.

\section{Appendix i: Gaussian Normal Coordinates}

In this appendix we give the proof of Lemma 1; i.e., that

$$
d s^{2}=d\left(w^{n}\right)^{2}+g_{i j} d w^{i} d w^{j},
$$

where $w$ denotes the Gaussian normal coordinate system constructed in the paragraph preceding Lemma $1 .{ }^{12}$ To verify (3.12) it suffices to show that

$$
\left\langle\frac{\partial}{\partial w^{i}}, \frac{\partial}{\partial w^{n}}\right\rangle=0
$$

for all $i \neq n$, where $\langle$,$\rangle denotes the inner product with respect to the metric g$. This is a consequence of the following well-known identities:

$$
\begin{gathered}
\frac{\partial}{\partial w^{n}}\left\langle\frac{\partial}{\partial w^{i}}, \frac{\partial}{\partial w^{j}}\right\rangle=\left\langle\nabla_{\frac{\partial}{\partial w^{n}}} \frac{\partial}{\partial w^{i}}, \frac{\partial}{\partial w^{j}}\right\rangle+\left\langle\frac{\partial}{\partial w^{i}}, \nabla_{\frac{\partial}{\partial w^{n}}} \frac{\partial}{\partial w^{j}}\right\rangle, \\
\nabla_{\boldsymbol{X}} \boldsymbol{Y}-\nabla_{\boldsymbol{X}} \boldsymbol{X}=[\boldsymbol{X}, \boldsymbol{Y}],
\end{gathered}
$$

where $\boldsymbol{X}$ and $\boldsymbol{Y}$ denote arbitrary smooth vector fields, $\nabla$ denotes the covariant derivative defined (in coordinates) by (2.7) and $[$,$] denotes the Lie bracket. Since$ by construction of Gaussian normal coordinates, $\frac{\partial}{\partial w^{n}}=\frac{d}{d s} \gamma_{P}(s)$ for some $P \in \Sigma$, this implies that $\frac{\partial}{\partial w^{n}}$ is parallel along the curves $\gamma$. Thus

$$
\nabla \frac{\partial}{\partial w^{n}} \frac{\partial}{\partial w^{n}}=0
$$

in the $w$-coordinate system, and (7.1) implies that

$$
\frac{\partial}{\partial w^{n}}\left\langle\frac{\partial}{\partial w^{n}}, \frac{\partial}{\partial w^{i}}\right\rangle=\left\langle\frac{\partial}{\partial w^{n}}, \nabla_{\frac{\partial}{\partial w^{n}}} \frac{\partial}{\partial w^{i}}\right\rangle \text {. }
$$

${ }^{12}$ This result is well known, [2]; we include it for completeness. 
From (7.2),

$$
\nabla \frac{\partial}{\partial w^{n}} \frac{\partial}{\partial w^{i}}=\nabla \frac{\partial}{\partial w^{i}} \frac{\partial}{\partial w^{n}}+\left[\frac{\partial}{\partial w^{n}}, \frac{\partial}{\partial w^{i}}\right]=\nabla \frac{\partial}{\partial w^{i}} \frac{\partial}{\partial w^{n}},
$$

because $\left\{\frac{\partial}{\partial w^{1}}, \ldots, \frac{\partial}{\partial w^{n}}\right\}$ are coordinate vector fields. Therefore, using (7.3) and (7.4) we have

$$
\frac{\partial}{\partial w^{n}}\left\langle\frac{\partial}{\partial w^{n}}, \frac{\partial}{\partial w^{i}}\right\rangle=\left\langle\frac{\partial}{\partial w^{n}}, \nabla \frac{\partial}{\partial w^{i}} \frac{\partial}{\partial w^{n}}\right\rangle=\frac{1}{2} \frac{\partial}{\partial w^{i}}\left\langle\frac{\partial}{\partial w^{n}}, \frac{\partial}{\partial w^{n}}\right\rangle 0 .
$$

Thus, $\frac{\partial}{\partial w^{n}}\left\langle\frac{\partial}{\partial w^{n}}, \frac{\partial}{\partial w^{i}}\right\rangle \equiv 0$ in $w$-coordinates, so $\left\langle\frac{\partial}{\partial w^{n}}, \frac{\partial}{\partial w^{i}}\right\rangle$ is identically equal to a constant, which must be zero because it is zero on $\Sigma$ by construction. This completes the proof of Lemma 2.

\section{Appendix ii: Proof of Proposition 1}

In this appendix we supply the proof of statements (4.50)-(4.54) of Proposition 1. The transformation $\Psi$ that maps the $(t, r)$-coordinates of the $\mathrm{R}-\mathrm{W}$ metric to the $(\bar{t}, \bar{r})$-coordinates of the O-T metric is given by

$$
\begin{gathered}
d \bar{r}=\dot{R} r d t+R d r \\
d \bar{t}=\psi C d t-\psi E d \bar{r}=(\psi C-\psi E \dot{R} r) d t-\psi E R d r
\end{gathered}
$$

where we have used (4.28) together with the fact that $\bar{r}=\Psi_{2}(t, r)=R(t) r$. From these it follows that

$$
\frac{\partial \bar{x}^{i}}{\partial x^{j}}=\left[\begin{array}{cc}
\psi C-\psi E \dot{R} r & -\psi E R \\
\dot{R} r & R
\end{array}\right]_{j}^{i},
$$

where in this section we use the notation $x=(t, r), \overline{\boldsymbol{x}}=(\bar{t}, \bar{r})$, and $\tilde{\boldsymbol{x}}=(t, \bar{r})$, and we suppress the $(\theta, \varphi)$ coordinates. (Here, the upper $i$ and lower $j$ on the right-hand side of (8.1) denotes the $(i, j)$-entry of the matrix.) From these relations it follows easily that

$$
\begin{aligned}
& \frac{\partial \tilde{\boldsymbol{x}}}{\partial \boldsymbol{x}}=\left[\begin{array}{cc}
1 & 0 \\
\dot{R} r & R
\end{array}\right]_{j}^{i}, \\
& \frac{\partial \tilde{\boldsymbol{x}}}{\partial \bar{x}}=\left[\begin{array}{cc}
\frac{1}{\psi \boldsymbol{C}} & \frac{E}{C} \\
0 & 1
\end{array}\right]_{j}^{i} .
\end{aligned}
$$

Now in the (tr)-coordinate plane, the R-W and I-S metrics have components $g_{R W}^{i j}$ and $g_{O T}^{i j}$ in $x$ - and $\bar{x}$-coordinates given respectively by

$$
\begin{gathered}
g_{R W}^{i j}=\left[\begin{array}{cc}
-0 & 0 \\
0 & \frac{R^{2}}{1-k r^{2}}
\end{array}\right]_{j}^{i}, \\
g_{O T}^{i j}=\left[\begin{array}{cr}
-B^{-1} & 0 \\
0 & A
\end{array}\right]_{j}^{i},
\end{gathered}
$$


where $A=1-2 \mathscr{G} M / \bar{r}$, and $B$ satisfies (4.8). Now on the shock surface $M=\frac{4 \pi}{3} \rho \bar{r}^{3}$, the metrics $g_{R W}$ and $g_{O T}$ agree, by which we mean that

$$
g_{R W}^{\alpha \beta}=\frac{\partial \boldsymbol{x}^{\alpha}}{\partial \overline{\boldsymbol{x}}^{i}} g_{O T}^{i j} \frac{\partial \boldsymbol{x}^{\beta}}{\partial \overline{\boldsymbol{x}}^{j}}
$$

Rather than calculate this out directly, we use the fact that the R-W and O-T metrics must have components that agree on the shock surface in the $\tilde{x}$-coordinates. Thus we calculate

$$
\begin{aligned}
& \tilde{g}_{R W}^{\alpha \beta}=\frac{\partial \tilde{\boldsymbol{x}}^{\alpha}}{\partial \boldsymbol{x}^{i}} g_{R W}^{i j} \frac{\partial \tilde{\boldsymbol{x}}^{\beta}}{\partial \boldsymbol{x}^{j}}=\left[\begin{array}{cc}
-1 & -\dot{R} r \\
-\dot{R} r & -\dot{R}^{2} r^{2}+\left(1-k r^{2}\right)
\end{array}\right]^{\alpha \beta}, \\
& \tilde{g}_{O T}^{\alpha \beta}=\frac{\partial \tilde{x}^{\alpha}}{\partial \overline{\boldsymbol{x}}^{i}} g_{I S}^{i j} \frac{\partial \tilde{\boldsymbol{x}}^{\beta}}{\partial \overline{\boldsymbol{x}}^{j}}=\left[\begin{array}{cc}
-\frac{1}{\psi^{2} C^{2} B}+\frac{A E^{2}}{C^{2}} & \frac{A E}{C} \\
\frac{A E}{C} & A
\end{array}\right]^{\alpha \beta} .
\end{aligned}
$$

(Again, the superscript $\alpha \beta$ on the right-hand side of (8.6) and (8.7) denotes the $(\alpha, \beta)$ entry of the matrix.) Equating the $(0,1)$-entries in $(8.6)$ and $(8.7)$ we obtain $(4.52)$. Equating the (1,1)-entries in (8.6) and (8.7) we obtain (4.54), and this together with (4.52) gives (4.53). Equating the (0,0)-entries in (8.6) and (8.7) gives the first equality in (4.50), and applying (4.54) gives the second. Finally, (4.51) follows from (4.52) together with (4.33), $E=-R \dot{R} r$. This concludes the proof of Proposition 1.

Alternatively, we can derive (4.50)-(4.54) directly from (4.5), (4.17) and (4.37), together with the expressions (4.31), (4.33) and (4.38) for $C, E$, and $B$, respectively. To obtain (4.54), solve (4.37) for $\rho$, solve (4.5) for $M$, and substitute these into (4.17). To obtain (4.51), multiply (4.17) by $r^{2}$, solve for $(8 \pi \mathscr{G} / 3) \rho R^{2} R^{2}$, and substitute this into (4.31). Using (4.51) together with (4.33) gives (4.52). The identity (4.52) together with (4.54) yields (4.53). Statement (4.38) together with (4.51) gives $1 / \psi^{2} C^{2}=(B / A)\left(1-k r^{2}\right)$. Using (4.51) together with (4.33) and (4.54), in the expression $1+A E^{2} / C^{2}$ gives the last equality in (4.50)).

Acknowledgements. We thank DeMETRI CHRISTODOULOU for his encouragement, his interest in our work, and for several stimulating conversations. The work of SMOLLER was supported in part by NSF Applied Mathematics Grant Number DMS-89-05205 and the Institute of Theoretical Dynamics, UC-Davis. The work of TEMPLE was supported in part by NSF Applied Mathematics Grant Number DMS-86-13450, and the Institute of Theoretical Dynamics, UC-Davis. B. TEMPLE thanks the University of Michigan for its hospitality during his sabbatical year 1991-92.

\section{References}

1. R. Courant \& K. O. Friedrichs, Supersonic Flow and Shock Waves, Wiley, 1948.

2. A. Gray, Tubes, Addison-Wesley, 1990.

3. W. ISRAEL, Singular hypersurfaces and thin shells in general relativity, Il Nuovo Cimento, 54B. (1966), 1-14. 
4. W. ISRAEL, Dark stars: the evolution of an idea, in: 300 years of gravitation, edited by S. W. HaWkING \& W. ISRAEL, Cambridge University Press, 1987, pp. 199-276.

5. P. D. LAX, Hyperbolic systems of conservation laws, $I$, Comm. Pure Appl. Math., 10 (1957), pp. 537-566.

6. G. C. MCVITTIE, Gravitational collapse to a small volume, Astro. Phys. J., 140 (1964), pp. $401-416$.

7. C. MISNER \& D. SHARP, Relativistic equations for adiabatic, spherically symmetric gravitational collapse, Phys. Rev., 26 (1964), pp. 571-576.

8. C. MISNER, K. THORNE \& J. WheELER, Gravitation, Freeman, 1973.

9. J. R. OPPENHEIMER \& J. R. SNYDER, On continued gravitational contraction, Phys. Rev., 56 (1939), pp. 455-459.

10. J. R. OpPenheimer \& G. M. Volkoff, On massive neutron cores, Phys. Rev., 55 (1939), pp. 374-381.

11. J. SMOLLER, Shock Waves and Reaction-Diffusion Equations, Springer-Verlag, 1983.

12. J. SMOller \& B. TemPle, Global solutions of the relativistic Euler equations, Comm. Math. Phys., 156 (1993), pp. 67-99.

13. R. Tolman, Relativity, Thermodynamics and Cosmology, Oxford University Press, 1934.

14. R. TOlman, Static solutions of Einstein's field equations for spheres of fluid, Phys. Rev., 55 (1939), pp. 364-374.

15. S. WeInBerg, Gravitation and Cosmology: Principles and Applications of the General Theory of Relativity, Wiley, 1972.

Department of Mathematics University of Michigan

Ann Arbor, Michigan 48109

and

Department of Mathematics University of California, Davis Davis, California 95616 